\title{
MSC-derived miR-181b Overexpressing Exosomes Enhanced Osteointegration by Enhancing M2 Polarization of Macrophages via Targeting the PRKCD/AKT Pathway
}

Wei Liu

Second Military Medical University

Muyu Yu

Shanghai Jiao Tong University

Feng Chen

Shanghai Jiao Tong University

Dong Xie

Peking University People's Hospital

\section{Longqing Wang}

East Region Military Command General Hospital

\section{Cheng Ye}

Second Military Medical University

Qi Zhu

Second Military Medical University

Mingzhe Shao

Shanghai Jiao Tong University

Lili Yang ( $\nabla$ yangll@smmu.edu.cn)

Second Military Medical University https://orcid.org/0000-0001-8764-2177

\section{Research}

Keywords: Mesenchymal stem cell, Macrophage polarization, MiR-181b, Exosome, Osteointegration, Inflammation

Posted Date: August 5th, 2020

DOI: https://doi.org/10.21203/rs.3.rs-52931/v1

License: (9) (1) This work is licensed under a Creative Commons Attribution 4.0 International License. Read Full License 


\section{Abstract}

Background: Many patients suffer from implant loosening after the implantation of titanium alloy caused by immune response to the foreign bodies and this could inhibit osteogenesis, which could possibly give rise to poor osteointegration and there is currently no appropriate solution in clinical practice. Exosomes overexpressing miRNA has been proven to be a suitable candidate for solving this problem. In this study, we explored whether miR-181b could exert beneficial effect on promoting M2 macrophage polarization, thus inhibiting inflammation as well as promoting osteogenesis and elaborated the underlying mechanism in vitro. Furthermore, we aimed to find whether exosomes overexpressing miR-181b (Exo181b) could enhance osteointegration in vivo.

Methods: In vitro and in vivo studies were carried out for assessing the anti-inflammatory and proosteogenesis effect of miR-181b. In vitro, ELISA was applied for the detection of the inflammation factors levels including IL-6, TNF-a, as well as IL-10 and the percentage of M1 or M2 polarization was determined by flow cytometry. Also, qRT-PCR was used for the detection of the relative gene expression of the CCR7, CD206, Arg-1, iNOS, VEGF and BMP-2 genes. Western blotting was applied for detecting the protein expression of PRKCD, AKT and p-AKT. In vivo, we established air pouch model for evaluating the effect of Exo-181b on macrophage polarization and distal femoral bone defect model was established for determining the osteointegration effect of Exo-181b by MicroCT, sequential fluorescent labeling and histological analysis.

Results: In vitro, we firstly verified that miR-181b significantly enhanced M2 polarization and inhibited inflammation by suppressing PRKCD and activating p-AKT. Then, in vivo, we verified that Exo-181b enhanced M2 polarization, reduced the inflammatory response and enhanced osteointegration.

Conclusions: MiR-181b could suppress inflammatory response by regulating the PRKCD/AKT signaling pathway and promoting M2 polarization, which further promoting osteogenesis of hBMSC in vitro and Exo-181b could promote osteointegration in vivo.

\section{Background}

At present, some patients suffer from implant loosening after receiving titanium alloy internal fixation or prosthetic implants due to the excessive innate immune response to the foreign bodies, which could give rise to an acute or serious inflammatory reaction around the internal fixation or prosthesis and impair the osteogenesis process in the medullary cavity, leading to poor osteointegration eventually [1]. On the contrary, an appropriate anti-inflammatory immune response is favorable for angiogenesis and osteogenesis [2, 3]. Therefore, we should aim to improve the local immune environment and promote the angiogenesis and osteogenesis that avail the osteointegration.

The development of osteoimmunology gives us an inspiration that we can properly modify the implants and modulate the local excessive inflammatory response to avoid undesirable osseointegration and improve the patients' quality of life after surgery [4]. The osteoimmunology concept indicates that the 
implant surface properties govern the effects (stimulation or inhibition) of macrophages exert on osteoblasts.

Macrophages play a key role in wound healing and tissue regeneration [5]. Macrophages contain classical activation (M1) and alternative activation (M2) subsets. M1 subtype promotes inflammation and is not conducive to tissue repair, while $\mathrm{M} 2$ inhibits inflammation and promotes tissue regeneration and repair. For example, zinc has been reported of promoting osteogenesis by promoting M2 macrophage polarization [6]. Also, hierarchical intrafibrillarly mineralized collagen material was fabricated for inducing M2 macrophage polarization for bone regeneration [7].

MicroRNA (miRNA) is one of the non-coding RNAs that can perform epigenetic function [8]. It could exert its effects by binding to the 3 ' untranslated region (UTR) of specific target mRNA for promoting the degradation of mRNA and/or inhibiting their translation [9]. Mir-181b could provide cardioprotection after acute myocardial infarction by inhibiting M1 macrophage polarization in an exosomal manner [10]. Also, by directly targeting Notch1, miR-181b could reduce atherosclerotic plaque vulnerability by enhancing M2 polarization. However, there is no research focusing on the immune regulation of miR-181b on titanium implants. Therefore, we desire to explore the role of miR-181b on osteoimmunology. Our aim is to apply miR-181b in vivo, but miRNAs are single-stranded RNAs which will be degraded easily by enzymes in vivo and cannot fully exert their effects. Therefore, a carrier is needed for protecting them from degradation.

Exosomes are extracellular nanoscale vesicles with a diameter from 30-200nm [11]. They carry complex cargos including proteins, nucleic acids, lipids and play an important role in intercellular communication [12]. Completely natural exosomes could not be sufficient for treating all kinds of diseases and some modifications need to be made [13]. Since miRNA is one of the cargos that could be used in tissue regeneration [14]. Thus, we could establish modularized extracellular vesicles by endowing the exosomes with specific miRNA via overexpression method by transfection [13]

MSCs are cells with immunomodulatory and multipotent abilities, which will be beneficial for inflammation-related diseases [15]. It has been reported that exosomes derived from hBMSC could regulate macrophage phenotypes and exert immunomodulatory effect because these two types of cells coexist in bone marrow and there exists cellular communication mediated by extracellular vesicles such as exosomes $[16,17]$. Furthermore, MSC-derived exosomes have been applied in many fields. For example, MSC-derived exosomes could regulate macrophage polarization through miR-182 and thus attenuating myocardial ischaemia reperfusion injury [18]. hUCMSC-derived exosome could attenuate burn-induced excessive inflammation by mediating miR-181c [19].

In this study, we aim to regulate macrophage polarization by delivering exosomes overexpressing miR181 and inhibiting the immune response caused by titanium alloy as well as promoting osteogenesis, thus promoting osteointegration and reducing the occurrence of implant loosening.

\section{Materials And Methods}




\section{The preparations of cells}

We obtained hBMSCs (P4) and RAW264.7 cells from Chinese Academy of Sciences. These two kinds of cells were respectively cultured in complete a-MEM and high glucose DMEM, which consists of $10 \%$ FBS (Gibco) and 1\% penicillin as well as streptomycin. We divided the experiment into following groups: PBS group, Ti group (titanium particle), Ti+miR-181bm NC group (titanium particle; NC: miR-181 b mimic negative control), Ti+miR-181 bm group (titanium particle, miR-181b mimic), Ti-miR-181bi NC (titanium particle, miR-181b inhibitor negative control), Ti-miR-181 bi (titanium particle, miR-181b inhibitor), siPRKCD (PRKCD that is inhibited by siRNA). Then, RAW264.7 was seeded into 6-well plates, treated with the above conditions and incubated in the environment of $37^{\circ} \mathrm{C}$ and $5 \% \mathrm{CO}_{2}$.

\section{The identification of hBMSCs}

For the characterization of hBMSCs, we obtained images of hBMSC by optical microscope for explore whether hBMSC could adhere to the plastic disk. Also, in vitro tri-lineage differentiation including adipogenesis, osteogenesis, chondrogenesis capacity were performed. Meanwhile, flow cytometry for detecting the surface markers of hBMSC was conducted. The adipogenesis (Oil Red $\mathrm{O}$ staining), osteogenesis (Alizarin Red staining), chondrogenesis (Alcian Blue staining) were assessed after being induced by osteoblast inducing medium for 3 weeks.

\section{The detection of inflammatory factors by ELISA}

After seeded into 24-well plates RAW264.7 cells were treated with PBS, Ti, Ti+miR-181bm NC and Ti+miR$181 \mathrm{bm}$ for $24 \mathrm{~h}$. Subsequently, the cell supernatants were acquired and used for measuring the concentrations of inflammatory and anti-inflammatory factors including TNF-a, IL-6 and IL-10 ELISA kit (Anogen, Canada).

\section{qRT-PCR analysis}

The relative gene expression including miR-181b, Arg-1, iNOS, CCR7, CD206, ALP, RUNX2, OCN, VEGF and BMP-2 was detected by qRT-PCR. Firstly, we applied TRIzol reagent (Invitrogen) for extracting the total RNA of RAW264.7 and hBMSC. Then, PrimeScript RT reagent Kit (Takara; Vazyme Biotech) was used for reverse transcription for acquiring complementary DNA of the extracted total RNA. Subsequently, qRT-PCR analysis experiments were conducted by SYBR Green detection reagent system (Takara). Finally, the levels of relative gene expression were calculated by applying the $2^{\wedge}-(\triangle \triangle C T)$ and 18S RNA levels was used for normalization.

\section{Cell proliferation and migration}

The proliferation of hBMSCs were determined by CCK- 8 assay. In short, $2 \times 10^{3}$ cells/well hBMSCs were seeded on 96-well plate. After cultured with equivalent conditioned culture medium extracted from PBS, Ti, Ti+miR-181 bm NC, Ti+miR-181 bm groups after 1, 3 and 7 days, we removed and replaced culture 
medium by fresh culture medium and CCK-8 solution (Dojindo, Japan). The absorbance was evaluated by ELISA plate reader (Epoch, BIO-TEK, USA) at $450 \mathrm{~nm}$ after $2 \mathrm{~h}$ at $37^{\circ} \mathrm{C}$.

Migration ability was assessed by transwell assay. Specifically, $1.5 \times 10^{4} \mathrm{hBMSC}$ were seeded on the upper chamber of a transwell plate with basal culture medium (Millipore). And the lower chamber was added with conditioned medium extracted from PBS, Ti, Ti+miR-181 bm NC, Ti+miR-181 bm groups. $24 \mathrm{~h}$ later, we removed the upper chamber cells, fixed the lower chamber cells with $4 \%$ paraformaldehyde and stained the migrated cells by $0.5 \%$ crystal violet for $5 \mathrm{~min}$. Optical microscope (Olympus IX 70 , Tokyo, Japan) was utilized for the observing the migrated cells before counted by Image $\mathrm{J}$.

\section{Osteogenic differentiation}

We evaluated the osteogenesis by Alkaline phosphatase (ALP) staining, Alizarin red staining and detecting the relative gene levels of ALP, RUNX2 (runt-related transcription factor 2), and OCN (osteocalcin), VEGF, BMP-2. Osteogenic differentiation medium supplemented with conditioned medium extracted from PBS, Ti, Ti+miR-181 bm NC, Ti+miR-181 bm groups for inducing hBMSCs for 14 and 21 days respectively. The cells induced by osteogenic differentiation medium were fixed with $4 \%$ paraformaldehyde. ALP (Beyotime) or Alizarin Red (Cyagen) staining solution were used for the staining. The samples were then observed and photographed by inverted optical microscope (Olympus IX 70, Tokyo, Japan). The relative gene levels including ALP, RUNX-2, OCN were assessed by qRT-PCR.

\section{The extraction and identification of exosome}

Exo and Exo-181b were acquired from the supernatant by ultracentrifugation. Concretely, miR-181bm NC and miR-181bm were used for the transfection of hBMSCs with serum-free culture medium for $72 \mathrm{~h}$. Then, we acquired and centrifuged the medium at $400 \mathrm{~g}$ for $6 \mathrm{~min}$ and 2,100 $\mathrm{g}$ for $25 \mathrm{~min}$ for discarding dead cells when the cell confluence reached about $80 \%$. Subsequently, a $0.22-\mu \mathrm{m}$ filter was used for the filtration of the supernatants for getting rid of the nanoscale non-exosome impurities (Micropore). After that, the supernatant was filtrated by Ultra-Clear ${ }^{\text {TM }}$ tubes (Beckman Coulter, USA) for filtrating at 100,000 $\mathrm{g}$ by ultracentrifuge for $1.5 \mathrm{~h}$ twice. Eventually, we resuspended the required pellets by PBS and kept them in $-80^{\circ} \mathrm{C}$ refrigerator.

Exosomes are nanoscale extracellular vesicles and transmission electron microscopy (TEM, JEM-1400) was applied for the observation of the ultrastructure and shape. In addition, nanoparticle tracking analysis (NTA, ZetaView PMX 110, Particle Metrix) was used for detecting the size distribution as well as nanoparticle concentration. Western blotting was utilized for the detection of the specific markers of exosomes CD9, Tsg101 and Alix.

\section{Air pouch model}

In our research, animal surgeries were approved by the Animal Care and Ethics Committee of Shanghai Sixth People's Hospital and were conducted in accordance with established guidelines. C57/BL6 mice 
were anesthetized by sodium pentobarbital and sterilized air was injected subcutaneously for establishing an air pouch model. Meanwhile, PBS, PT, PT+Exo and PT+Exo-181b were implanted into subcutaneous tissue for detecting the effect of Exo-181b on inflammation. After four days, we washed subcutaneous pouch by $3 \mathrm{ml}$ stain buffer for collecting cells. Then, the percentage of M1 and M2 macrophages was determined by flow cytometry.

\section{The establishment of femoral bone defect model of rats.}

Porous titanium alloy in our research has a pore size from 500 to $600 \mu \mathrm{m}$, which is too large for the sustained release of exosomes. Therefore, we applied a biocompatible, biodegradable commercial hyaluronic acid hydrogel for solving this problem. Thirty-two rats (12-week, male) were randomly divided into four groups containing porous titanium alloy group (PT), PT+HA hydrogel (PT-H), PT-H+exosomes group (PT-H-Exo), Ti-H-Exo+miR-181b group (PT-H-Exo-181b) $(n=8)$. After anesthetizing the rats by $0.6 \%$ phenobarbital sodium at a dose of $1 \mathrm{ml} / \mathrm{kg}$ intraperitoneally, we made a knee medial incision and exposed the medial of femur condyles by moving the patella outward. Then, femoral condyles were drilled laterally with a 3.5-mm diameter trephine (Nouvag AG) to produce 5-mm-deep bone defect and implanted with PT, PT-H, PT-H-Exo and PT-H-Exo-181b. After that, the soft tissues were sutured layer by layer with 4-0 resorbable sutures postoperatively. When the anesthesia was finished, all the rats underwent operation were returned to the biosafety facility and guaranteed enough food and water. Each rat received antibiotics intramuscularly for 3 days. The rats were euthanized 3 months after the surgery and the femurs were harvested and fixed in $4 \%$ paraformaldehyde solution.

\section{MicroCT}

MicroCT (Skyscan 1176, Kontich, Belgium) was utilized for measuring new bone formation. The samples were scanned and the resolution was $18 \mu \mathrm{m}$. Afterwards, TV (total volume), BV (bone volume), BV/TV as well as BMD (bone mineral density) in the defects were assessed by CTAn image analysis software. Sagittal and three-dimensional reconstruction images were performed by Dataviewer and CTVox.

\section{Sequential fluorescent labeling}

Trichromatic fluorescent labeling was used for assessing the new bone formation. Specifically, $25 \mathrm{mg} / \mathrm{kg}$ tetracycline (TE, Sigma, yellow), $30 \mathrm{mg} / \mathrm{kg}$ alizarin reds (AL, Sigma, red) and $20 \mathrm{mg} / \mathrm{kg}$ calcein (CA, Sigma,green) were injected intraperitoneally respectively after 3, 6 and 9 weeks postoperatively.

\section{Histological analysis}

We dehydrated the harvested samples by gradient concentrations of alcohols and were immersed in xylene for transparency for 4 hours. Later, the samples were immersed in the infiltration liquid and embedding liquid for 2 days. Subsequently, all the samples were put into glass bottle full of embedding liquid for polymerization in $37^{\circ} \mathrm{C}$ water bath. Then, we applied microtome for acquiring undecalcificated

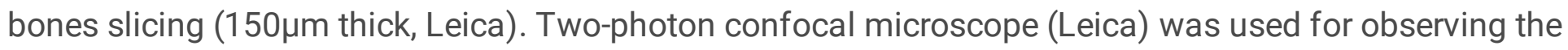
fluorescent labeling. The excitation/emission wavelengths for tetracycline, alizarin red and calcein were 
405/560-590 nm, 543/580-670 nm, and 488/500-550 nm respectively. Later, the sections were polished to $50 \mu \mathrm{m}$ and were stained with van Gieson's picrofuchsin for evaluating the new bone formation. Finally, the quantification of new bone formation area was calculated by Image $\mathrm{J}$.

\section{Bioinformation analysis}

Four different databases including TargetScan, miRanda, miRDB and PicTar were used for predicting the target genes of miR-181b binding sites. Then we applied gene ontology database and chose the targets that were related to inflammatory responses. All the genes were intersected by Venn chart for obtaining the common genes.

\section{Luciferase report}

We purchased luciferase reporter plasmids containing the 3'-UTRs of PRKCD and the corresponding mutated 3'-UTR. After that, we inserted the 3'-UTRs and mutated 3'-UTR into pRL-CMV reporter vector (Promega, USA). Then, 96-well plates were used for the incubation of 293T cells for $24 \mathrm{~h}$. After that, a reporter plasmid and miR-181b mimics using Lipofectamine 2000 (11668-019, Invitrogen) was cotransfected into each well. Subsequently, we detected renilla and firefly luciferase activities through DualLuciferase Reporter Assay System (E1910, Promega).

\section{Western blotting}

Western blotting for the evaluation of PRKCD, AKT and p-AKT protein expression. Prechilled RIPA buffer was applied for lysing and harvesting the protein of RAW264.7 on ice. Subsequently, $5 \times$ loading buffer was applied for diluting the lysates. Then, we boiled the dilution at $95^{\circ} \mathrm{C}$ for about $5 \mathrm{~min}$. Then, SDS-PAGE was used for separating different by molecular weight and the separated proteins were transferred onto a PVDF membrane (Merck-Millipore) and blocked with nonfat milk. Eventually, the primary antibodies and secondary antibodies were applied for incubating PVDF membrane overnight and for $1 \mathrm{~h}$ respectively. An ECL substrate kit was applied for visualizing the protein bands of the PVDF membrane.

\section{Statistics}

We used Student-Newman-Keuls post hoc tests, one-way ANOVA and two-way ANOVA for evaluating the statistical significance. Statistical analysis of the mean \pm SEM was performed by Graphpad. $P$ value $<$ 0.05 was deemed significant.

\section{Results}

\section{The identification of hBMSC}

Adherence ability, tri-lineage differentiation capability as well as surface markers are deemed the basic criteria for identifying hBMSC and were performed in our research (Figure 1). Firstly, hBMSCs has been prove with the ability to adhere to the plastic culture disk (Figure 1a). Secondly, pluripotency was 
evaluated by trilineage differentiation, which contained adipogenesis, osteogenesis and chondrogenesis. The hBMSC showed abundant lipid droplet, calcium nodules, polysaccharides and proteoglycans by Oil Red, Alizarin Red and Alcian Blue staining respectively (Figure 1b). Thirdly, surface markers including CD73, CD90 and CD105 showed $>95 \%$ positive and CD 45 and CD34 showed $<2 \%$ negative of hBMSCs, meeting the demand of the basic criteria for the identification of hBMSC (Figure 1c).

\section{MiR-181b inhibited the inflammation by enhancing M2 polarization in vitro.}

TNF-a, IL-6, CCR7 and iNOS are the markers of the inflammatory state of M1 polarization, while IL-10, CD206, Arg-1 represents the anti-inflammatory state of M2 polarization. Therefore, for determining the macrophage polarization state after treated with PBS, Ti, Ti-miR-181bm NC and Ti-miR-181bm, we tested $\mathrm{M} 1$ and M2 polarization markers after incubated for $24 \mathrm{~h}$ by ELISA and qRT-PCR. By ELISA detection, we found that IL- 6 as well as TNF-a secretion (inflammatory markers) in the Ti-miR-181bm group was significantly decrease compared with those in the PBS, Ti and Ti-miR-181bm NC groups. Meanwhile, it can be observed that anti-inflammatory markers IL-10 secretion in the Ti-miR-181bm group was significantly increased compared with those in the PBS, Ti and Ti-miR-181bm NC groups (Figure 2a). By qRT-PCR, the relative levels of M1 polarization markers CCR7 and iNOS in the Ti-miR-181bm was significantly decreased compared with those in the PBS, Ti and Ti-miR-181 bm NC groups. Also, it can be observed that Arg-1 and IL-10 gene expression of in the Ti-miR-181 bm group was significantly enhanced compared with those in the PBS, Ti and Ti-miR-181 bm NC groups (Figure 2b). By flow cytometry, CCR7 was high in the Ti group but significantly reduced after treated with miR-181 bm (Figure 2c, e). CD206 was low in the Ti group but significantly increased with the treatment of miR-181b (Figure 2d, f).

\section{MiR-181b promotes the proliferation, migration and osteogenic differentiation abilities of hBMSCs.}

From the above results, we could know that miR-181b could promote M2 polarization, which was reported of immunomodulatory and pro-osteogenesis ability by increasing the gene expression and secretion of BMP-2 and VEGF from RAW264.7 [6]. Therefore, we collected the conditioned medium from RAW264.7 treated with PBS, Ti, Ti-miR-181bm NC and Ti-miR-181bm and explored their effect on osteogenesis. As we can observe, miR-181 bm promoted the proliferation of hBMSCs evaluated by CCK-8 assay (Figure 3a). Migration ability is also an important property of cells for osteointegration. Thus, Transwell assay was utilized, illustrating that miR-181b significantly enhanced the migration ability of hBMSC in comparison with the other groups, especially the Ti group (Figure $3 b$ ). Furthermore, as for the osteogenic differentiation, ALP and alizarin red staining (ARS) showed that Exo-181b have the most ALP expression and mineral deposition in comparison with PBS, Ti and Ti-miR-181 bm NC groups ((Figure 3b).

To further quantify osteogenesis-related genes ALP, RUNX2 and OCN influenced by miR-181b, qRT-PCR was carried out. It can be seen that the relative gene expression of ALP, RUNX2 and OCN genes were significantly enhanced at day 14 in Ti-miR-181bm group compared with PBS, Ti and Ti-miR-181bm NC groups, indicating that miR-181b exerts an important effect on osteogenesis (Figure 3c). For explaining the reason why the conditioned medium from miR-181b-treated RAW264.7 supertanants, we determined the relative expression of osteogenesis-related genes BMP-2 and VEGF, which showed a significant 
increase in comparison with the other groups (Figure 3d). The above data together demonstrated that miR-181b could exert immunomodulatory and further promote osteogenesis of hBMSC in vitro.

\section{The isolation and characterization ofhBMSC-derived exosomes}

For further verification of the role of miR-181b on macrophage polarization in vivo, exosome was utilized as a carrier for protecting and delivering miR-181b. TEM, NTA and Western blotting were utilized for the assessment of the characterization of isolated particles. TEM pictures revealed the round bilayer lipid membrane vesicles of the two groups (Figure 4a) and NTA test showed that Exo and Exo-181b displayed similar peak diameters at approximately 130nm (Figure 4b). Meanwhile, western blotting demonstrated that exosomes markers Alix, Tsg101, and CD9 high expression in the isolated particles with no significant difference between the two groups (Figure 4c). The expression of miR-181b was remarkably higher in hBMSC treated with miR-181 bm than miR-181 bm NC while Exo-181b had more miR-181b than Exo (Figure 4d). The above results confirmed the successful isolation of exosomes and the overexpression of miR-181b in hBMSC and hBMSC-extracted exosomes.

\section{Exo-181b inhibited the inflammation by enhancing M2 polarization in vivo.}

It is known that titanium alloy could cause inflammatory response by promoting M1 polarization in vivo. Therefore, we applied C57BL/ 6 for establishing air pouch model for assessing the effect of Exo-181b on promoting M2 polarization in vivo by flow cytometry. CCR7 representing M1 phenotype was high in the PT group but significantly reduced treated with Exo-181b (Figure 5a, c). CD206, the marker of M2 polarization, has low positive percentage in the PT group but significantly increased with the treatment of Exo-181b (Figure 5b, d). From these results, we can conclude that Exo-181b could enhance M2 polarization in vivo.

\section{Exo-181b incorporated by hydrogel improved the osseointegration between PT and bone in femoral defect model.}

\section{MicroCT analysis}

We established femoral bone defect model for assessing the osteointegration effect of PT-H-Exo-181b in vivo. By microCT analysis, we could observe the newly formed bone by sagittal and 3D reconstruction images. It can be seen that the newly formed bone area in PT-H-Exo-181b group was significantly more than that of PT, PT-H, PT-H-Exo groups (Figure 6a). TV means the total volume of the region of interest. MicroCT quantitative analysis illustrated no significant difference of TV among PT, PT-H, PT-H-Exo, PT-HExo-181b groups. BV means the bone volume of the region of interest and BV/TV reflects the percentage of bone tissue of the total tissue. As for BV and BV/TV, microCT analysis illustrated that PT-H-Exo-181b showed increased trend in comparison with the other groups. BMD means the bone mineral density, which reflects the deposition of minerals. Quantitative analysis showed that PT-H-Exo-181b significantly increased BMD, which means much more mineral deposition (Figure 6b). TV, BV, BV/TV and BMD 
collectively showed the osteogenesis condition around the implant and PT-H-Exo-181b possessed better pro-osteogenesis and pro-osteointegration abilities.

\section{Fluorochrome labeling and histology analysis}

For assessing osteointegration, undecalcificated bones slicing was prepared. New bone formation was analyzed after 3, 6 and 9 weeks (Figure 7).

Three weeks later, the percentage of TE labeling area of the PT-H-Exo-181b group was more than that in the PT, PT-H and PT-H-Exo groups. Six weeks later, the percentage of AR labeling in the PT-H-Exo-181 b scaffold groups was markedly higher than that in PT, PT-H and PT-H-Exo groups. After 9 weeks, PT-H-Exo$181 \mathrm{~b}$ group gained the highest percentage of CA labeling area and there were also significant differences among PT, PT-H and PT-H-Exo groups (Figure 7a, c).

Van Gieson (VG) solution applied for new bone formation staining consists of picric acid and acid fuchsin. The new bone formation showed red color after stained by VG solution. For the analysis of van Gieson staining, we can find that the PT-H-Exo-181b group showed significantly more new bone formation than that in PT group, PT-H group and PT-H-Exo group. As for the quantitative analysis, we applied Image $\mathrm{J}$ for calculating the area of new bone formation, PT-H-Exo-181b group also exhibited significantly more new bone formation that the other three groups, suggesting a better osteointegration effect (Figure 7b,d).

\section{MiR-181b enhanced M2 polarization by inhibiting the PRKCD/AKT signaling pathway.}

For searching for the potential targets of miR-181b, we applied bioinformation tools. Four databases including TargetScan, miRanda, miRDB and PicTar were chosen for finding the potential targets. By Venn chart, we combined the common 20 targets among the databases. Then, we searched gene ontology database to find 2270 genes related to inflammatory response. By combining the results of the common 20 targets and 2270 genes from gene ontology, we finally got the 1 potential target PRKCD (Figure 8a).

For further verifying the direct inhibition of miR-181b on PRKCD, luciferase assay was applied. The results showed that PRKCD-Wt was inhibited after treated with miR-181b. However, after the mutation of the $3^{\prime} U T R$ of the PRKCD, the inhibition effect was significantly weakened, suggesting that PRKCD was a direct target of miR-181b (Figure 8b). To explore the underlying mechanisms of miR-181b on antiinflammatory effect, we detected the protein expression level of PRKCD and the phosphorylation level of AKT, which were reported of playing an important role in promoting M2 macrophage polarization in vitro $[20,21]$. In our results, the phosphorylation of AKT in the Ti group was suppressed but was significantly rescued by miR-181b, which verified the enhancement of $M 2$ polarization. PRKCD, negatively regulating the phosphorylation levels of AKT and thus inhibiting M2 polarization, was activated in Ti group but was suppressed in miR-181b group, showing the enhancement of M2 polarization (Figure 8c).

The inhibition of PRKCD enhanced M2 macrophage polarization by inhibiting the PRKCD/AKT signaling pathway. 
For further proving the role of miR-181b and PRKCD in M2 macrophage polarization, miR-181b inhibitor and siRNA were applied. In our results, the protein level of PRKCD was reduced and the phosphorylation of AKT was significantly enhanced after treated with si-PRKCD. However, the protein level of PRKCD was significantly increased and the phosphorylation of AKT was reduced after treated with miR-181bi, which further verified the effect of miR-181b on PRKCD and p-AKT (Figure 9a).

Furthermore, we found that the levels IL- 6 as well as TNF-a in the miR-181bi+si-PRKCD group were significantly increased and the secretion of anti-inflammatory markers IL-10 was decreased compared with those in the miR-181bi NC+si-PRKCD group. Meanwhile, the levels IL- 6 and TNF-a in the miR$181 \mathrm{bi}+$ si-PRKCD group were significantly reduced and the secretion of IL-10 was significantly increased compared with those in the miR-181bi+si-PRKCD NC group (Figure 9b).

By qRT-PCR, the relative levels of M1 polarization markers CCR7 and iNOS in the miR-181 bi+si-PRKCD group were significantly increased and M2 polarization markers CD206 and Arg-1 were significantly decreased compared with those in the miR-181 bi NC+si-PRKCD group. Also, it can be found that CCR7 and iNOS in the miR-181 bi+si-PRKCD group were significantly reduced and CD206 and Arg-1 were significantly increased compared with those of the miR-181bi NC+si-PRKCD group (Figure 9c).

By flow cytometry, CCR7 was high in the PT group but significantly reduced after treated with miR-181bm (Figure 9d, f). CD206 has a low percentage in the PT group but significantly increased with the treatment of miR-181b (Figure 9e, g).

\section{Discussion}

In this study, miR-181b promoted M2 macrophage polarization in vitro of RAW264.7, whose supernatant was then used for the culture of hBMSCs for the verification of osteogenic differentiation. In vivo, the exosomes overexpressing miR-181b was incorporated into hydrogel as a coating for the titanium alloy, which was implanted into the bone defect in rats and verified to significantly promote the osseointegration.

In clinical practices, when the titanium alloy was implanted into the bone, the normal bone tissue will be damaged, which will trigger a series of physiological reactions such as inflammatory response, bone formation and bone remodeling. The implants will be immersed in an environment containing different hemocytes, proteins and inflammatory factors [22]. The main inflammatory response of the host is haematoma as well as the activation of the immune system [23-26] Meanwhile, the monocytes in blood vessels will migrate to the implanting site, differentiate into macrophages and attach to the titanium alloy, recruiting other inflammatory cells by secreting IL-8\MCP-1 $\triangle \mathrm{MIP}-1 \beta$ and so on [22]. A blood clot will be formed after the implantation and the titanium alloy is exposed to the inflammatory cytokines. Once the inflammation response is weakened, microcapillaries will be formed inside the implants, which bring about vascularization and osteoblasts will be recruited around the implants. And osteoblasts will differentiate into woven bone a few weeks later [1]. Moderate inflammation is tolerable but some patients suffer from acute and uncontrolled inflammatory response, which could cause implant loosening duo the 
impediment of vessel invasion, formation and osteogenesis [27]. Therefore, it is of great importance to inhibit this improper inflammatory response.

M1 and M2 macrophage polarization are closely correlated with inflammation [28, 29]. Inhibition of M1 and enhancement of $\mathrm{M} 2$ will ameliorate inflammation and promote the transition from inflammatory stage to the regeneration stage. miR-181b derived and transferred from cardiosphere cells to macrophages could provide cardioprotective effects for the patients after acute myocardial infarction [10]. By shifting macrophage to M2 polarization state, miR-181b improves insulin sensitivity by the regulation of endothelial function [30]. In addition, the atherosclerotic plaque vulnerability was reduced by promoting M2 macrophage phenotypes by miR-181b [31]. Meanwhile, M2 phenotype exert great importance on tissue repair and remodeling [32]. Therefore, miR-181b with the ability to promote M2 polarization for inhibiting inflammation and enhance tissue regeneration is suitable for the application of osteoimmunology and osteointegration.

MiRNA has been widely studied as a main participant in gene therapy for bone research. Chi Yang et al. reported that miR-21 could promote osteogenesis via activating the PTEN/PI3K/Akt/HIF-1a pathway and BMSCs/ $\beta$-tricalcium phosphate modified by miR-21 enhanced the bone regeneration in calvarial defect model [33]. Lan Zhang demonstrated that overexpression of miR-335-5p enhanced the expression of proosteogenesis proteins RUNX2 and Osx, while downregulating the negative regulation factor DKK1 protein, thus promoting calvarial bone defect repair [34]. Yan Li reported that bone regeneration, the highly vascularized tissue, relies on the coupling between osteogenesis and angiogenesis, while the interruption of this coupling will cause bad effects to the bone regeneration. Therefore, they applied miR-26a for positively regulating angiogenic-osteogenic coupling and promote the calvarial defect regeneration [35].

For studying the potential mechanism of miR-181b on promoting M2 macrophage, we predicted that PRKCD was the downstream target of miR-181b. Since AKT is related to macrophage polarization, $\mathrm{PRKCD} / \mathrm{AKT}$ signaling pathway was studied. Akt, which is composed of three serine/threonine protein kinases including Akt1, Akt2, and Akt3, has been regarded as a key protein involved in macrophage polarization [36, 37]. Since the inhibition of Akt abrogates the upregulation of $M 2$ related genes, the activation of Akt is essential for M2 polarization [38]. Feng Liu et, al reported that TGF- $\beta$ secreted from MSC promoted RAW264.7 to M2 phenotype [39]. Guohua Wang et, al found that increased expression of glycogen synthase kinase 3 beta (GSK3 $\beta$ ) inhibited the activation of phosphatase and tensin homologue (PTEN), thus enhancing PI3K/Akt pathway, further enhancing microglia to M2 polarization [40]. Protein kinase $C$ (PKC) play an important role in many cell functions, such as proliferation, and differentiation and so on [41, 42]. PRKCD, namely PKCס, is one of the isozymes of PKC and is reported of inhibiting the phosphorylation of AKT in macrophages [20,42, 43]. Our results showed that miR-181b significantly downregulated the gene and protein expression of PRKCD, thereby enhancing p-AKT and polarizing macrophages to M2 phenotype. After using si-PRKCD, p-AKT was enhanced and the inflammation was weakened. Meanwhile, the application of miR-181b inhibitor showed the opposite trends that PRKCD was enhanced and the phosphorylation of AKT was suppressed, further verifying the role of miR-181b on M2 polarization. 
For delivering specific miRNA to specific targets, suitable nano carries are required in regenerative medicine [44]. Nanocarriers including viral vector and nonviral vector have been widely explored for effectively delivering specific cargos to the specific sites [45]. However, the application of these vectors remains a challenge because of some shortcomings such as poor cytocompatibility. Therefore, it is vital for seeking alternative nanocarriers for this crux. Fortunately, exosomes are natural derived nanocarriers secreted by host cells and endocytosed by recipient cells via fusing with the cellular membrane easily. Exosomes will avoid the potential cytotoxicity of other vectors and potential immunological rejection, becoming our first choice [46].

In our research, miR-181b could not only promote the polarization state of RAW264.7, but also indirectly promote osteogenesis. Our results demonstrated that the relative expression of OCN, RUNX2, ALP was enhanced and ALP as well as Alizarin red staining showed more alkaline phosphatase and mineralized nodules in miR-181b group. By qRT-PCR, we found the increased expression of pro-osteogenesis genes BMP-2 and VEGF in RAW264.7, which explains the osteogenic differentiation role of miR-181b on hBMSC and osteointegration between the implants and bone. It has been reported that human amniotic mesenchymal stromal cells (HAMSC) showed increased secretion of BMP-2 and VEGF by promoting the macrophages to M2 polarization and the osteogenesis of hBMSC was enhanced via co-culture method in a paracrine manner of HAMSC [47]. Epigallocatechin-3-gallate (EGCG) modified collagen was reported to promote the transition of macrophage to $\mathrm{M} 2$ polarization, thus inhibiting inflammatory response and increased the secretion of BMP-2 and VEGF, which further facilitates the osteogenesis by upregulating RUNX2 and OPN and promotes the new bone formation in vivo [48].

\section{Conclusion}

To sum up, our research verified that miR-181b could suppress inflammatory response by regulating the PRKCD/AKT signaling pathway and facilitating M2 polarization, which further improving osteogenesis in vitro and Exo-181b could promote osteointegration in vivo.

\section{Abbreviations}

Ti: titanium particles; miR-181bm:miR-181b mimic; NC: negative control; PT: porous titanium alloy; $\mathrm{H}$ : hydrogel; TE: tetracycline; AL: alizarin red; CA: calcein; Wt: wild type; Mt: mutant; miR-181bi: miR-181b inhibitor; si-PRKCD: PRKCD that is inhibited by siRNA.

\section{Declarations}

\section{Supplementary information}

The data can be seen in Supplementary material.

\section{Acknowledgements}


Not applicable.

\section{Authors' contributions}

WL: Formal analysis, Writing-original draft, Conceptualization, Data curation. MYY: Formal analysis. FC: Material fabrication. DX: Formal analysis. LQW: Data analysis. CY: Animal experiment assist. QZ: Animal experiment assist. MZS: Supervision. LLY: Supervision, Funding acquisition, Project design, Experiment instruction.

\section{Funding:}

This study was supported by the National Natural Science Foundation of China (NO. 81572194). Excellent Academic Leaders Training Program of the Shanghai Municipal Health and Family Planning System (NO.2017BR030), Science and Technology Innovation Action Plan of the Science and Technology Commission of Shanghai Municipality (NO. 17441900600).

\section{Availability of data and materials}

The datasets used and/or analyzed during the current study are available from the corresponding author on reasonable request.

\section{Ethics approval and consent to participate}

All animal procedures were approved by the Animal Research Committee of Shanghai Sixth People's Hospital affiliated to Shanghai Jiao Tong University.

\section{Consent for publication}

Not applicable.

\section{Competing interests}

The authors declare no competing interests.

\section{References}

1. Kohli N, Ho S, Brown SJ, Sawadkar P, Sharma V, Snow M, García-Gareta E. Bone remodelling in vitro: Where are we headed?: -A review on the current understanding of physiological bone remodelling and inflammation and the strategies for testing biomaterials in vitro. Bone. 2018;110:38-46. doi:10.1016/j.bone.2018.01.015.

2. Claes $L$, Recknagel $S$, Ignatius $A$. Fracture healing under healthy and inflammatory conditions. Nat Rev Rheumatol. 2012;8:133-43. doi:10.1038/nrrheum.2012.1.

3. Mokarram N, Bellamkonda RV. A perspective on immunomodulation and tissue repair. Ann Biomed Eng. 2014;42:338-51. doi:10.1007/s10439-013-0941-0. 
4. Tsukasaki $\mathrm{M}$, Takayanagi $\mathrm{H}$. Osteoimmunology: evolving concepts in bone-immune interactions in health and disease. Nat Rev Immunol. 2019;19:626-42. doi:10.1038/s41577-019-0178-8.

5. Wynn TA, Vannella KM. Macrophages in Tissue Repair, Regeneration, and Fibrosis. Immunity. 2016;44:450-62. doi:10.1016/j.immuni.2016.02.015.

6. Liu W, Li J, Cheng M, Wang Q, Yeung K, Chu PK, Zhang X. Zinc-Modified Sulfonated Polyetheretherketone Surface with Immunomodulatory Function for Guiding Cell Fate and Bone Regeneration. Adv Sci (Weinh). 2018;5:1800749. doi:10.1002/advs.201800749.

7. Jin SS, He DQ, Luo D, Wang Y, Yu M, Guan B, Fu Y, Li ZX, Zhang T, Zhou YH, Wang CY, Liu Y. A Biomimetic Hierarchical Nanointerface Orchestrates Macrophage Polarization and Mesenchymal Stem Cell Recruitment To Promote Endogenous Bone Regeneration. ACS Nano. 2019;13:6581-95. doi:10.1021/acsnano.9b00489.

8. Wei JW, Huang K, Yang C, Kang CS. Non-coding RNAs as regulators in epigenetics (Review). Oncol Rep. 2017;37:3-9. doi:10.3892/or.2016.5236.

9. Zhang B, Zhang Z, Li L, Qin YR, Liu H, Jiang C, Zeng TT, Li MQ, Xie D, Li Y, Guan XY, Zhu YH. TSPAN15 interacts with BTRC to promote oesophageal squamous cell carcinoma metastasis via activating NF-KB signaling. Nat Commun. 2018;9:1423. doi:10.1038/s41467-018-03716-9.

10. de Couto G, Gallet R, Cambier L, Jaghatspanyan E, Makkar N, Dawkins JF, Berman BP, Marbán E. Exosomal MicroRNA Transfer Into Macrophages Mediates Cellular Postconditioning. Circulation. 2017;136:200-14. doi:10.1161/CIRCULATIONAHA.116.024590.

11. Pegtel DM, Gould SJ. Exosomes. Annu Rev Biochem. 2019;88:487-514. doi:10.1146/annurevbiochem-013118-111902.

12. Tao SC, Guo SC, Li M, Ke QF, Guo YP, Zhang CQ. Chitosan Wound Dressings Incorporating Exosomes Derived from MicroRNA-126-Overexpressing Synovium Mesenchymal Stem Cells Provide Sustained Release of Exosomes and Heal Full-Thickness Skin Defects in a Diabetic Rat Model. Stem Cells Transl Med. 2017;6:736-47. doi:10.5966/sctm.2016-0275.

13. Tao SC, Guo SC, Zhang CQ. Modularized Extracellular Vesicles: The Dawn of Prospective Personalized and Precision Medicine. Adv Sci (Weinh). 2018. 5(2): 1700449.

14. Lai RC, Yeo RW, Lim SK. Mesenchymal stem cell exosomes. Semin Cell Dev Biol. 2015;40:82-8. doi:10.1016/j.semcdb.2015.03.001.

15. Zheng G, Huang R, Qiu G, Ge M, Wang J, Shu Q, Xu J. Mesenchymal stromal cell-derived extracellular vesicles: regenerative and immunomodulatory effects and potential applications in sepsis. Cell Tissue Res. 2018;374:1-15. doi:10.1007/s00441-018-2871-5.

16. Jafarzadeh N, Safari Z, Pornour M, Amirizadeh N, Forouzandeh Moghadam M, Sadeghizadeh M. Alteration of cellular and immune-related properties of bone marrow mesenchymal stem cells and macrophages by K562 chronic myeloid leukemia cell derived exosomes. J Cell Physiol. 2019;234:3697-710. doi:10.1002/jcp.27142.

17. Ekström K, Omar O, Granéli C, Wang X, Vazirisani F, Thomsen P. Monocyte exosomes stimulate the osteogenic gene expression of mesenchymal stem cells. PLoS One. 2013;8:e75227. 
doi:10.1371/journal.pone.0075227.

18. Zhao J, Li X, Hu J, Chen F, Qiao S, Sun X, Gao L, Xie J, Xu B. Mesenchymal stromal cell-derived exosomes attenuate myocardial ischaemia-reperfusion injury through miR-182-regulated macrophage polarization. Cardiovasc Res. 2019;115:1205-16. doi:10.1093/cvr/cvz040.

19. Li X, Liu L, Yang J, Yu Y, Chai J, Wang L, Ma L, Yin H. Exosome Derived From Human Umbilical Cord Mesenchymal Stem Cell Mediates MiR-181c Attenuating Burn-induced Excessive Inflammation. EBioMedicine. 2016;8:72-82. doi:10.1016/j.ebiom.2016.04.030.

20. Li Q, Park K, Xia Y, Matsumoto M, Qi W, Fu J, Yokomizo H, Khamaisi M, Wang X, Rask-Madsen C, King GL. Regulation of Macrophage Apoptosis and Atherosclerosis by Lipid-Induced PKCס Isoform Activation. Circ Res. 2017;121:1153-67. doi:10.1161/CIRCRESAHA.117.311606.

21. Covarrubias AJ, Aksoylar HI, Horng T. Control of macrophage metabolism and activation by mTOR and Akt signaling. Semin Immunol. 2015;27:286-96. doi:10.1016/j.smim.2015.08.001.

22. Franz S, Rammelt S, Scharnweber D, Simon JC. Immune responses to implants - a review of the implications for the design of immunomodulatory biomaterials. Biomaterials. 2011;32:6692-709. doi:10.1016/j.biomaterials.2011.05.078.

23. Nuss $\mathrm{KM}$, von Rechenberg B. Biocompatibility issues with modern implants in bone - a review for clinical orthopedics. Open Orthop J. 2008;2:66-78. doi:10.2174/1874325000802010066.

24. Muschler GF, Nakamoto C, Griffith LG. Engineering principles of clinical cell-based tissue engineering. J Bone Joint Surg Am. 2004;86:1541-58. doi:10.2106/00004623-200407000-00029.

25. Z Chen TK, RZ Murray RC, Chang J. Osteoimmunomodulation for the development of advanced bone biomaterials. Materials today. 2016.

26. MA Woodruff $C L, J$ Reichert $A B$, Chen F. Bone tissue engineering: from bench to bedside. Materials Today. 2012.

27. Miron RJ, Bosshardt DD. OsteoMacs: Key players around bone biomaterials. Biomaterials. 2016;82:119. doi:10.1016/j.biomaterials.2015.12.017.

28. Ying H, Kang Y, Zhang H, Zhao D, Xia J, Lu Z, Wang H, Xu F, Shi L. MiR-127 modulates macrophage polarization and promotes lung inflammation and injury by activating the JNK pathway. $\mathrm{J}$ Immunol. 2015;194:1239-51. doi:10.4049/jimmunol.1402088.

29. Olefsky JM, Glass CK. Macrophages, inflammation, and insulin resistance. Annu Rev Physiol. 2010;72:219-46. doi:10.1146/annurev-physiol-021909-135846.

30. Sun X, Lin J, Zhang Y, Kang S, Belkin N, Wara AK, Icli B, Hamburg NM, Li D, Feinberg MW. MicroRNA181b Improves Glucose Homeostasis and Insulin Sensitivity by Regulating Endothelial Function in White Adipose Tissue. Circ Res. 2016;118:810-21. doi:10.1161/CIRCRESAHA.115.308166.

31. An TH, He QW, Xia YP, Chen SC, Baral S, Mao L, Jin HJ, Li YN, Wang MD, Chen JG, Zhu LQ, Hu B. MiR$181 \mathrm{~b}$ Antagonizes Atherosclerotic Plaque Vulnerability Through Modulating Macrophage Polarization by Directly Targeting Notch1. Mol Neurobiol. 2017;54:6329-41. doi:10.1007/s12035-0160163-1. 
32. Mantovani A, Biswas SK, Galdiero MR, Sica A, Locati M. Macrophage plasticity and polarization in tissue repair and remodelling. J Pathol. 2013;229:176-85. doi:10.1002/path.4133.

33. Yang C, Liu X, Zhao K, Zhu Y, Hu B, Zhou Y, Wang M, Wu Y, Zhang C, Xu J, Ning Y, Zou D. miRNA-21 promotes osteogenesis via the PTEN/PI3K/Akt/HIF-1a pathway and enhances bone regeneration in critical size defects. Stem Cell Res Ther. 2019;10:65. doi:10.1186/s13287-019-1168-2.

34. Zhang L, Tang Y, Zhu X, Tu T, Sui L, Han Q, et al. Overexpression of MiR-335-5p Promotes Bone Formation and Regeneration in Mice. J Bone Miner Res. 2017;32:2466-75. doi:10.1002/jbmr.3230.

35. Li Y, Fan L, Liu S, Liu W, Zhang H, Zhou T, Wu D, Yang P, Shen L, Chen J, Jin Y. The promotion of bone regeneration through positive regulation of angiogenic-osteogenic coupling using microRNA-26a. Biomaterials. 2013;34:5048-58. doi:10.1016/j.biomaterials.2013.03.052.

36. Arranz A, Doxaki C, Vergadi E, Martinez de la Torre Y, Vaporidi K, Lagoudaki ED, leronymaki E, Androulidaki A, Venihaki M, Margioris AN, Stathopoulos EN, Tsichlis PN, Tsatsanis C. Akt1 and Akt2 protein kinases differentially contribute to macrophage polarization. Proc Natl Acad Sci U S A. 2012;109:9517-22. doi:10.1073/pnas.1119038109.

37. Liu L, Zhu X, Zhao T, Yu Y, Xue Y, Zou H. Sirt1 ameliorates monosodium urate crystal-induced inflammation by altering macrophage polarization via the PI3K/Akt/STAT6 pathway. Rheumatology (Oxford). 2019;58:1674-83. doi:10.1093/rheumatology/kez165.

38. Vergadi E, leronymaki E, Lyroni K, Vaporidi K, Tsatsanis C. Akt Signaling Pathway in Macrophage Activation and M1/M2 Polarization. J Immunol. 2017. 198(3): 1006-1014.

39. Liu F, Qiu H, Xue M, et al. MSC-secreted TGF- $\beta$ regulates lipopolysaccharide-stimulated macrophage M2-like polarization via the Akt/Fox01 pathway. Stem Cell Res Ther. 2019. 10(1): 345.

40. Wang G, Shi Y, Jiang X, Leak RK, Hu X, Wu Y, Pu H, Li WW, Tang B, Wang Y, Gao Y, Zheng P, Bennett MV, Chen J. HDAC inhibition prevents white matter injury by modulating microglia/macrophage polarization through the GSK3ß/PTEN/Akt axis. Proc Natl Acad Sci U S A. 2015;112:2853-8. doi:10.1073/pnas.1501441112.

41. Newton $A C$. Regulation of the $A B C$ kinases by phosphorylation: protein kinase $C$ as a paradigm. Biochem J. 2003;370:361-71. doi:10.1042/BJ20021626.

42. Li L, Sampat K, Hu N, Zakari J, Yuspa SH. Protein kinase C negatively regulates Akt activity and modifies UVC-induced apoptosis in mouse keratinocytes. J Biol Chem. 2006;281:3237-43. doi:10.1074/jbc.M512167200.

43. Naruse K, Rask-Madsen C, Takahara N, Ha SW, Suzuma K, Way KJ, Jacobs JR, Clermont AC, Ueki K, Ohshiro Y, Zhang J, Goldfine AB, King GL. Activation of vascular protein kinase C-beta inhibits Aktdependent endothelial nitric oxide synthase function in obesity-associated insulin resistance. Diabetes. 2006;55:691-8. doi:10.2337/diabetes.55.03.06.db05-0771.

44. Pina S, Oliveira JM, Reis RL. Natural-based nanocomposites for bone tissue engineering and regenerative medicine: a review. Adv Mater. 2015;27:1143-69. doi:10.1002/adma.201403354.

45. György B, Hung ME, Breakefield XO, Leonard JN. Therapeutic applications of extracellular vesicles: clinical promise and open questions. Annu Rev Pharmacol Toxicol. 2015;55:439-64. 
doi:10.1146/annurev-pharmtox-010814-124630.

46. Sun D, Zhuang X, Xiang X, Liu Y, Zhang S, Liu C, Barnes S, Grizzle W, Miller D, Zhang HG. A novel nanoparticle drug delivery system: the anti-inflammatory activity of curcumin is enhanced when encapsulated in exosomes. Mol Ther. 2010;18:1606-14. doi:10.1038/mt.2010.105.

47. Jiang F, Zhang W, Zhou M, Zhou Z, Shen M, Chen N, Jiang X. Human amniotic mesenchymal stromal cells promote bone regeneration via activating endogenous regeneration. Theranostics. 2020;10:6216-30. doi:10.7150/thno.45249.

48. Chu C, Wang Y, Wang Y, Yang R, Liu L, Rung S, Xiang L, Wu Y, Du S, Man Y, Qu Y. Evaluation of epigallocatechin-3-gallate (EGCG) modified collagen in guided bone regeneration (GBR) surgery and modulation of macrophage phenotype. Mater Sci Eng C Mater Biol Appl. 2019;99:73-82. doi:10.1016/j.msec.2019.01.083.

\section{Figures}

a

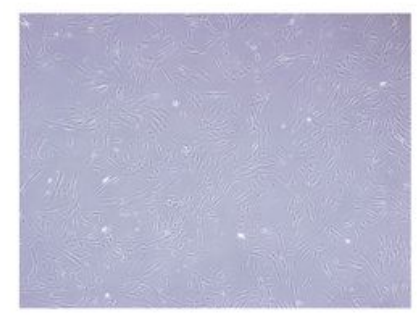

b

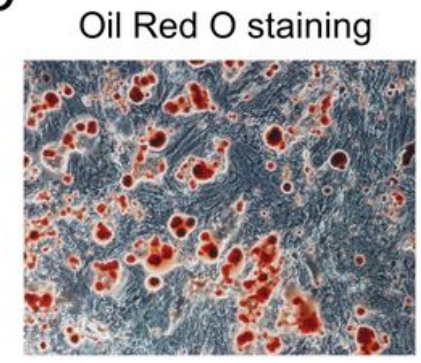

Alizarin Red staining

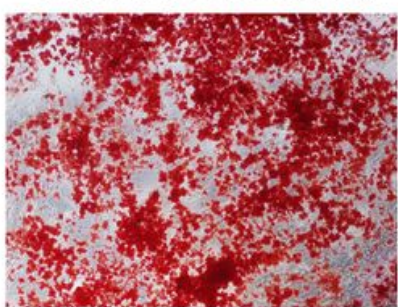

Alcian Blue staining

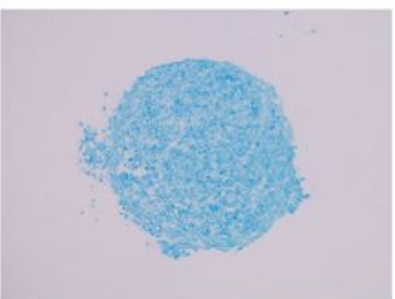

\section{C}
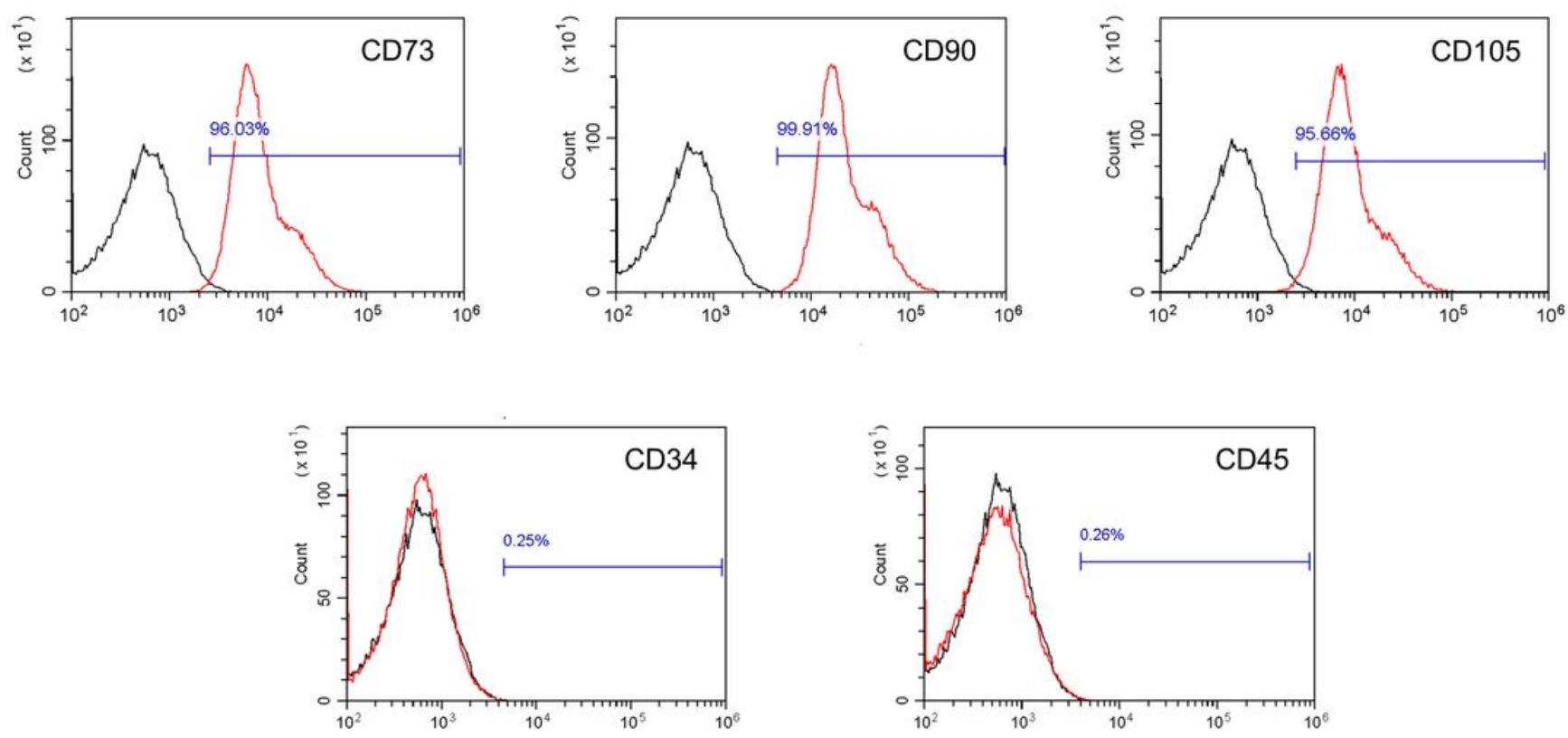

Figure 1 
The identification of hBMSC. a The verification of the adherence ability to plastic culture vessels of hBMSCs. Scale bar $=500 \mu \mathrm{m}$. b The tri-lineage differentiation ability of adipogenesis, osteogenesis, as well as chondrogenesis of hBMSC respectively. Scale bars $=100 \mu \mathrm{m}, 200 \mu \mathrm{m}$, and $200 \mu \mathrm{m}$ respectively. c The specific surface markers CD34, CD 45, CD73, CD90, CD105 of hBMSC by flow cytometry.

a
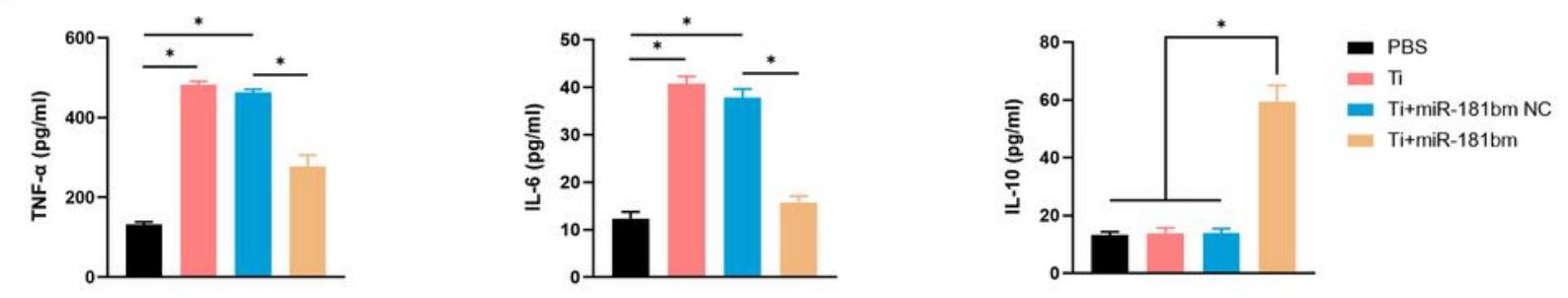

b
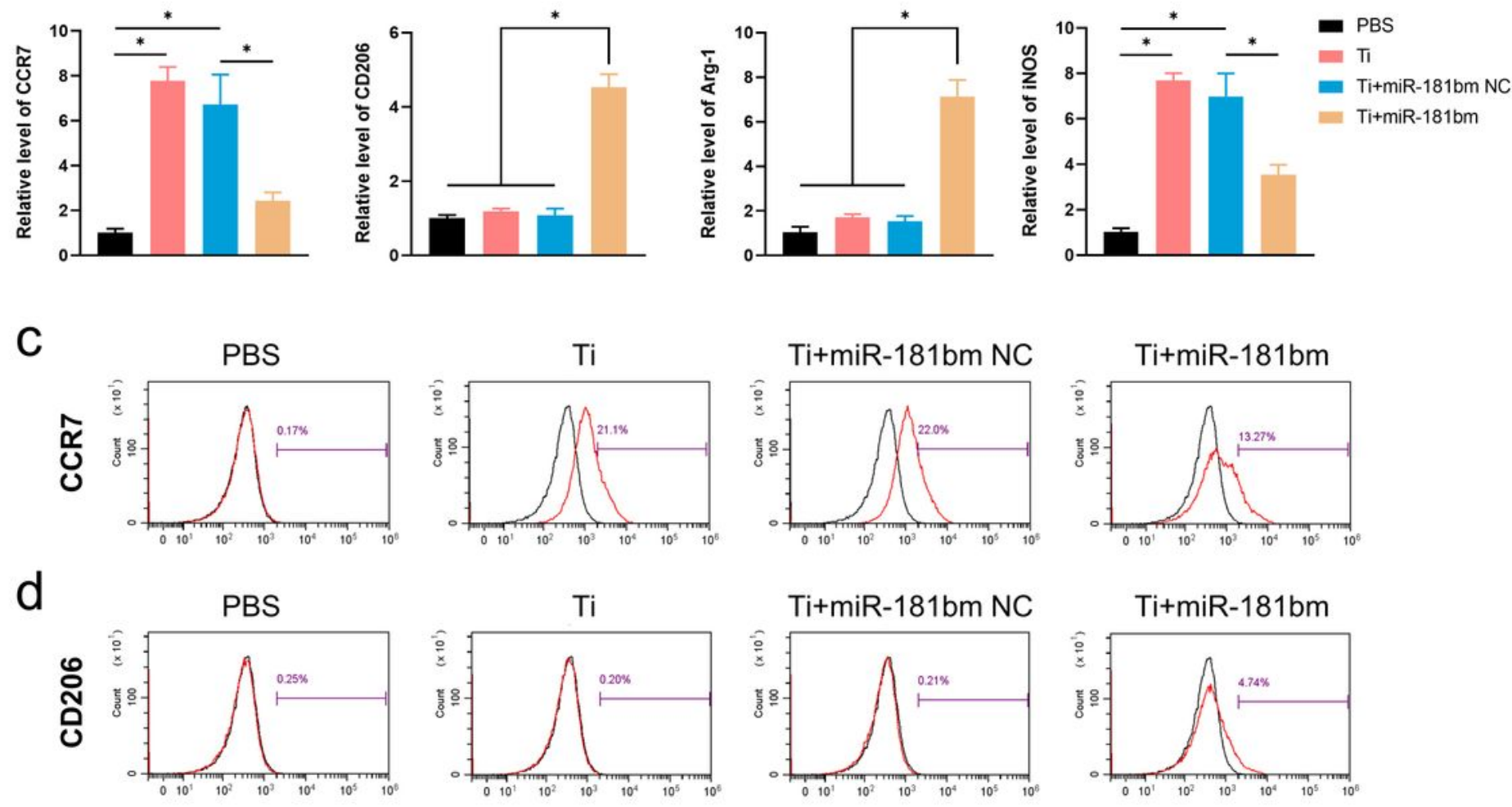

e
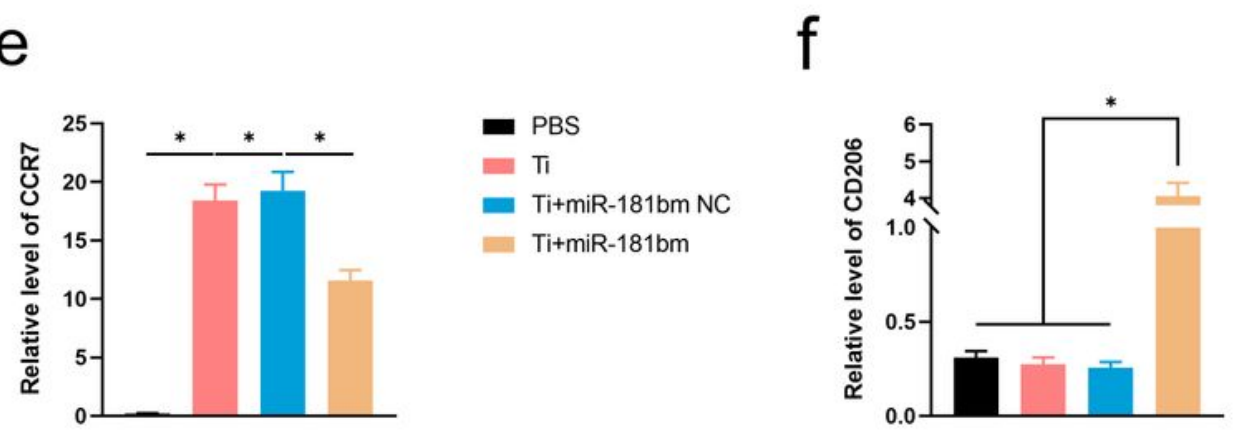

Figure 2 
MiR-181b inhibited the inflammatory response by enhancing M2 polarization macrophages in vitro. a The concentrations of TNF- $a$, IL-6, IL-10 of the supernatants detected by ELISA. $b$ The relative gene expression of CCR7, CD206, Arg-1 and iNOS was detected via qRT-PCR. c-d The percentage of CCR7 and CD206 positive cells verified by flow cytometry. e-f The quantitative analysis of the percentage of CCR7 and CD206 positive cells $(n=3, * p<0.05)$.

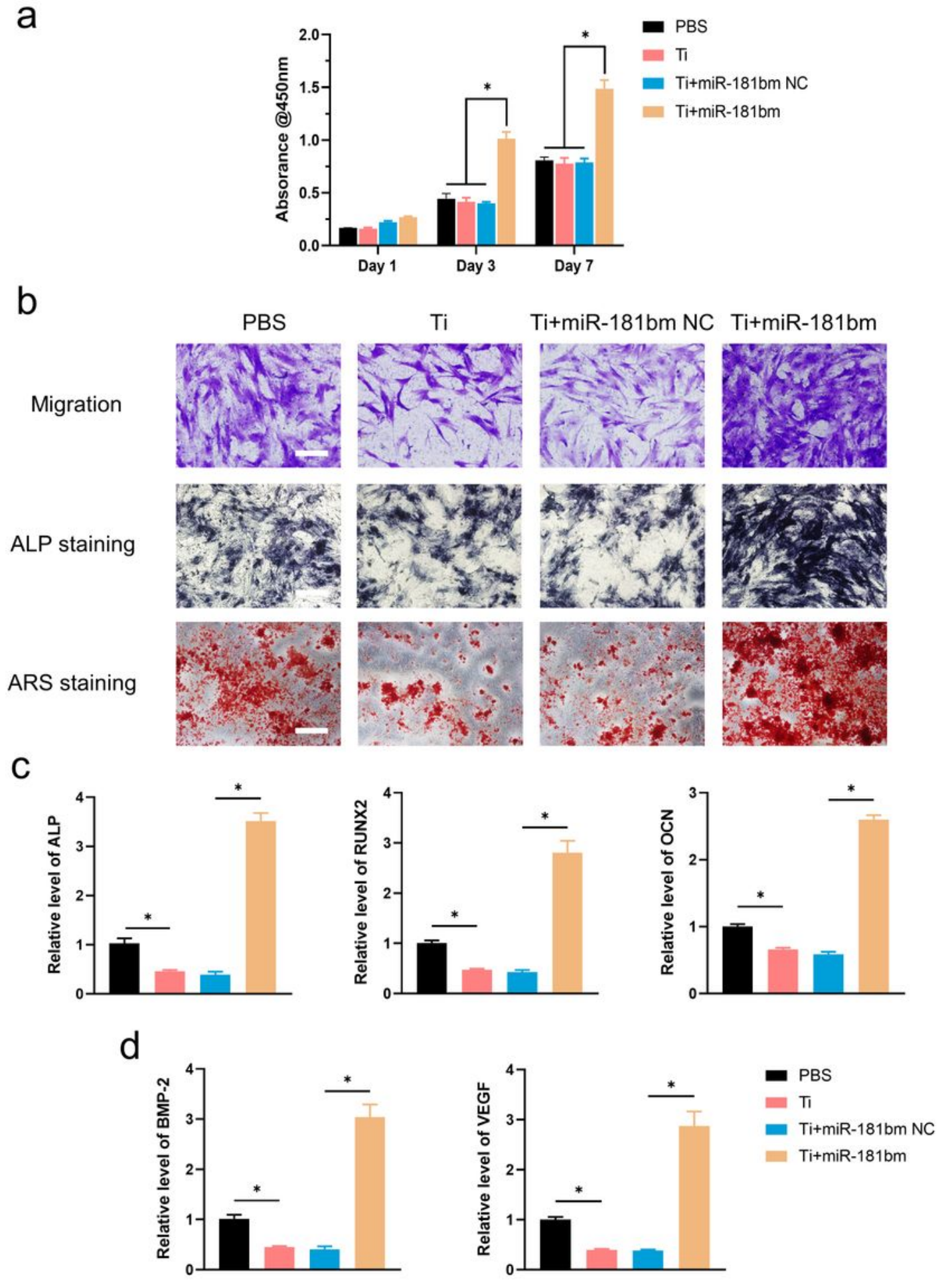

Figure 3 
Conditioned medium treated by miR-181b enhanced the proliferation, migration and osteogenic differentiation of hBMSCs in vitro. a The detection of proliferation of hBMSCs incubated with conditioned medium treated by PBS, Ti, Ti+miR-181b-NC and Ti+miR-181b by CCK-8 assay for 1, 3 and 7 days. $b$ Cell migration ability of hBMSCs incubated with conditioned medium treated by PBS, Ti, Ti+miR-181b-NC and Ti+miR-181b by transwell assay. Scale bar $=100 \mu \mathrm{m}$. ALP staining on day 14 and ARS on day 21 for the evaluation of osteogenic differentiation of hBMSCs. Scale bar=200 $\mu \mathrm{m}$. c Relative mRNA expression of qRT-PCR analysis for ALP, RUNX2, OCN of hBMSCs treated with conditioned medium treated by PBS, Ti, Ti+miR-181b-NC and Ti+miR-181b. d Relative mRNA expression of qRT-PCR analysis for VEGF and BMP2 of RAW264.7 treated with PBS, Ti, Ti+miR-181b-NC and Ti+miR-181b $\left(n=3,{ }^{*} p<0.05\right)$. 
a

Exo

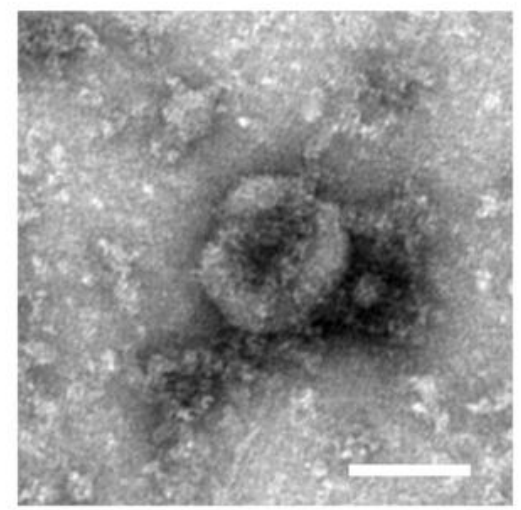

b

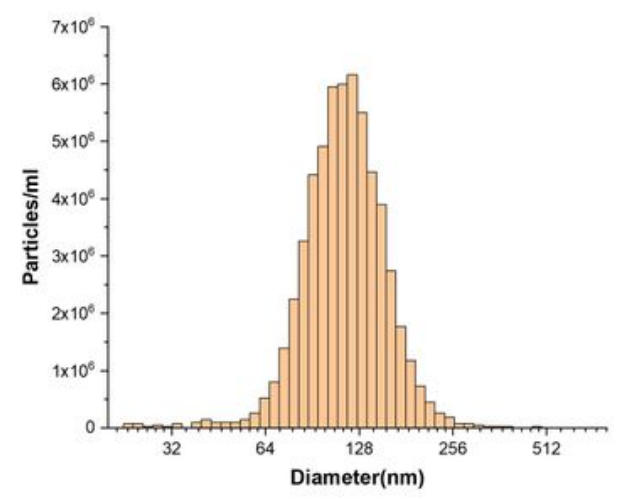

Exo-181b
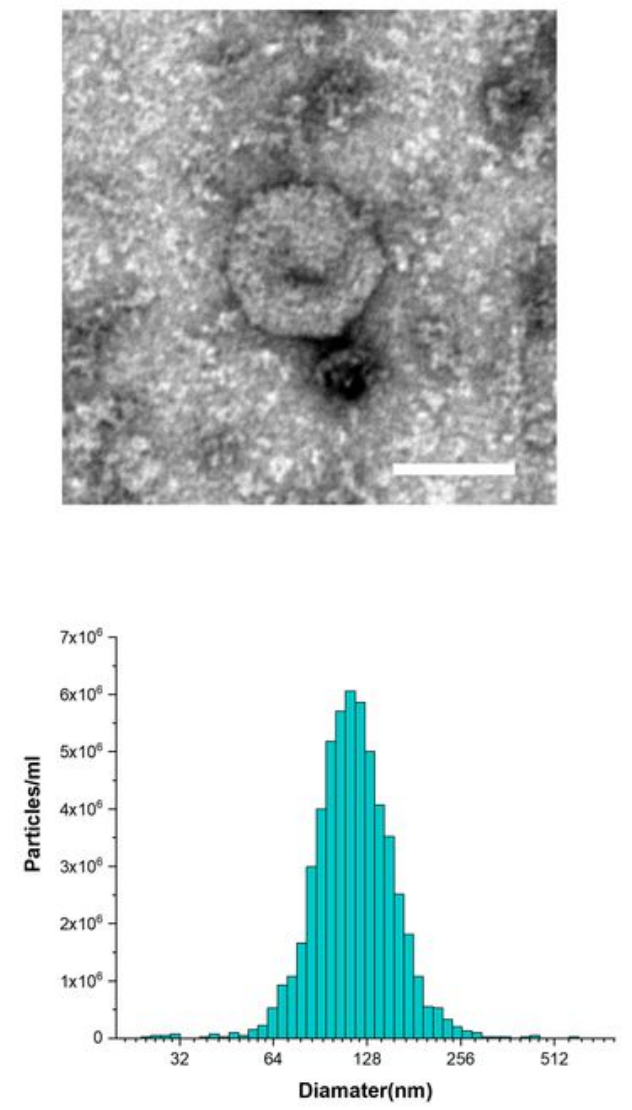

C

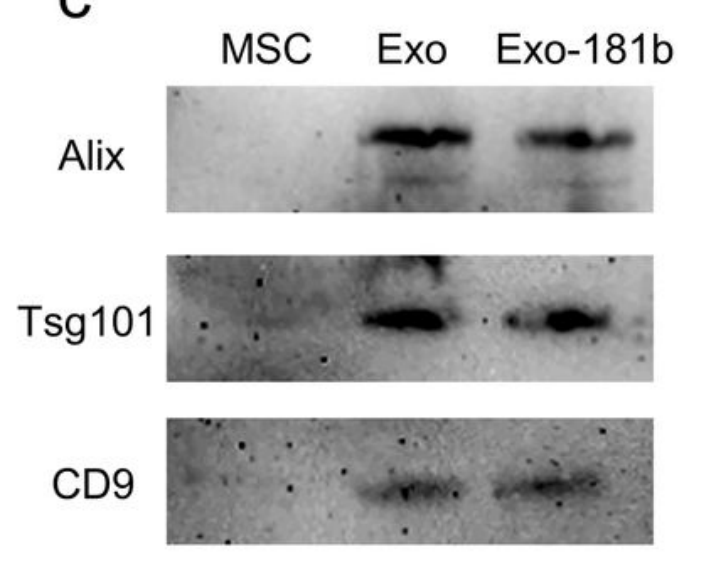

d

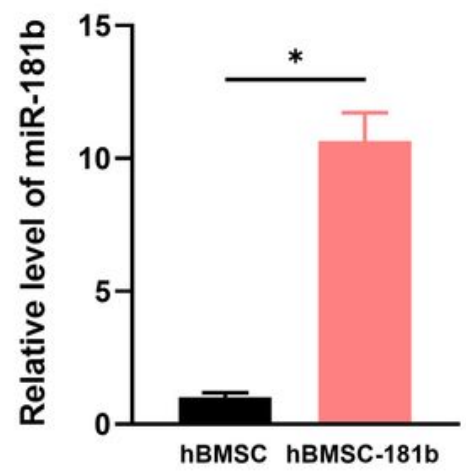

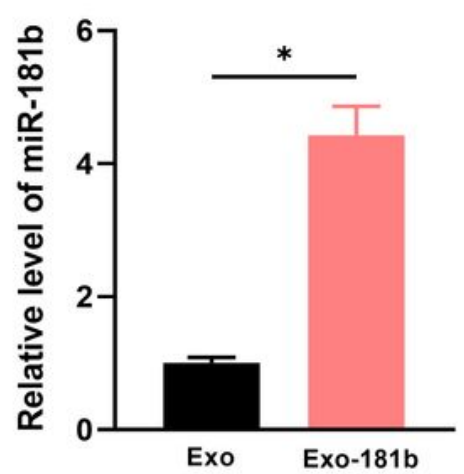

\section{Figure 4}

The identification of Exo and Exo-181b. a TEM was utilized for the observation of the morphology of Exo and Exo-181b. Scale bar=100 nm. b NTA analysis was used for analyzing the diameter distribution of Exo and Exo-181b. c The identification of Exo and Exo-181b by detecting their surface positive markers (CD9, Alix, and Tsg101) by western blot. $d$ The gene expression of miR-181b in hBMSC and exosome overexpressing miR-181b after treated by miR-181b mimic $\left(n=3,{ }^{*}<<0.05\right)$. 

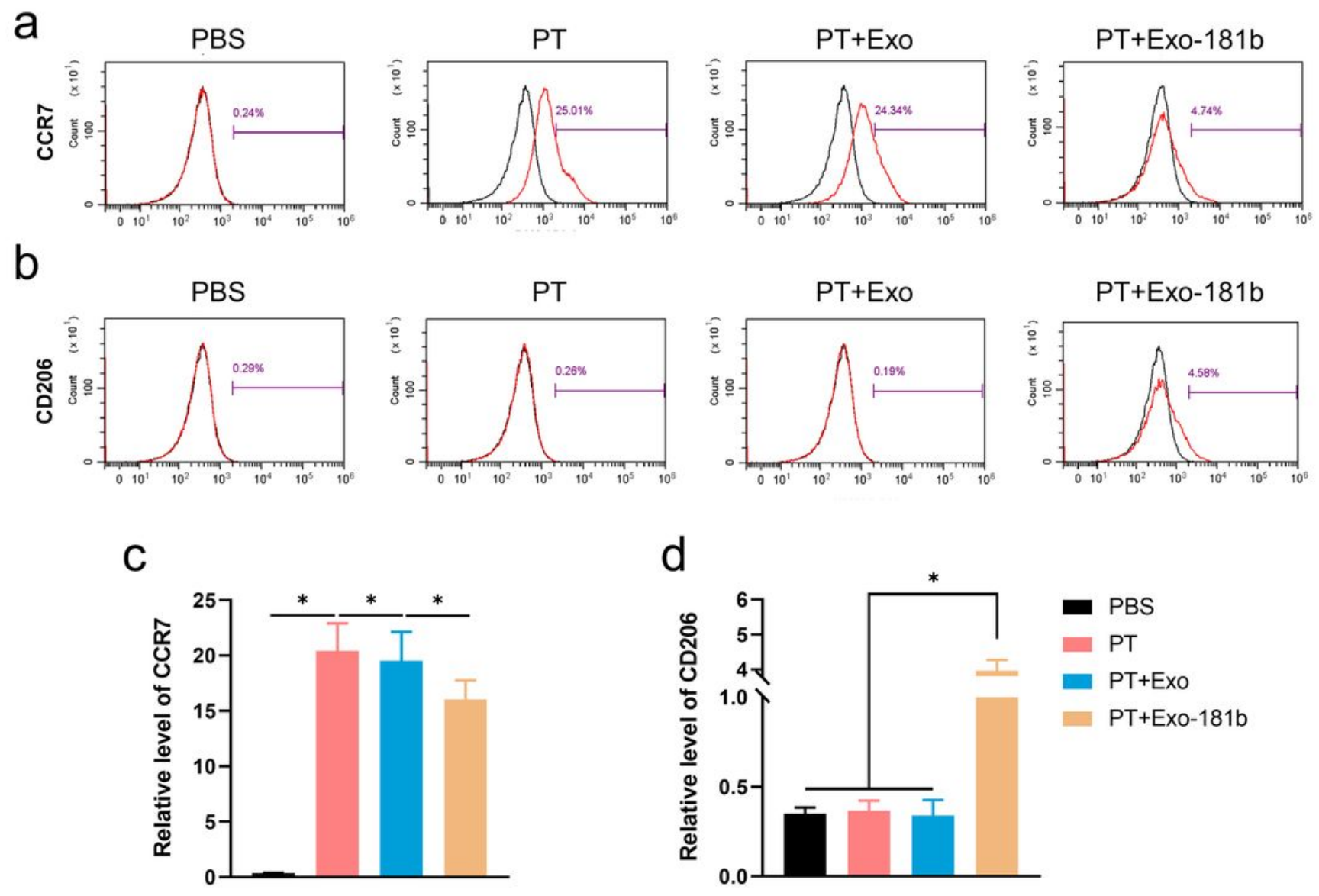

\section{Figure 5}

Exo-181b increased the percentage of M2 polarization macrophages in vivo. $a-b$ The percentage of CCR7 and CD206 of air pouch model treated by PBS, PT, PT +Exo, PT+Exo-181b by flow cytometry. c-d The corresponding quantification analysis of CCR7 and CD206 positive cells of flow cytometry $\left(n=3,{ }^{*}<0.05\right)$. 


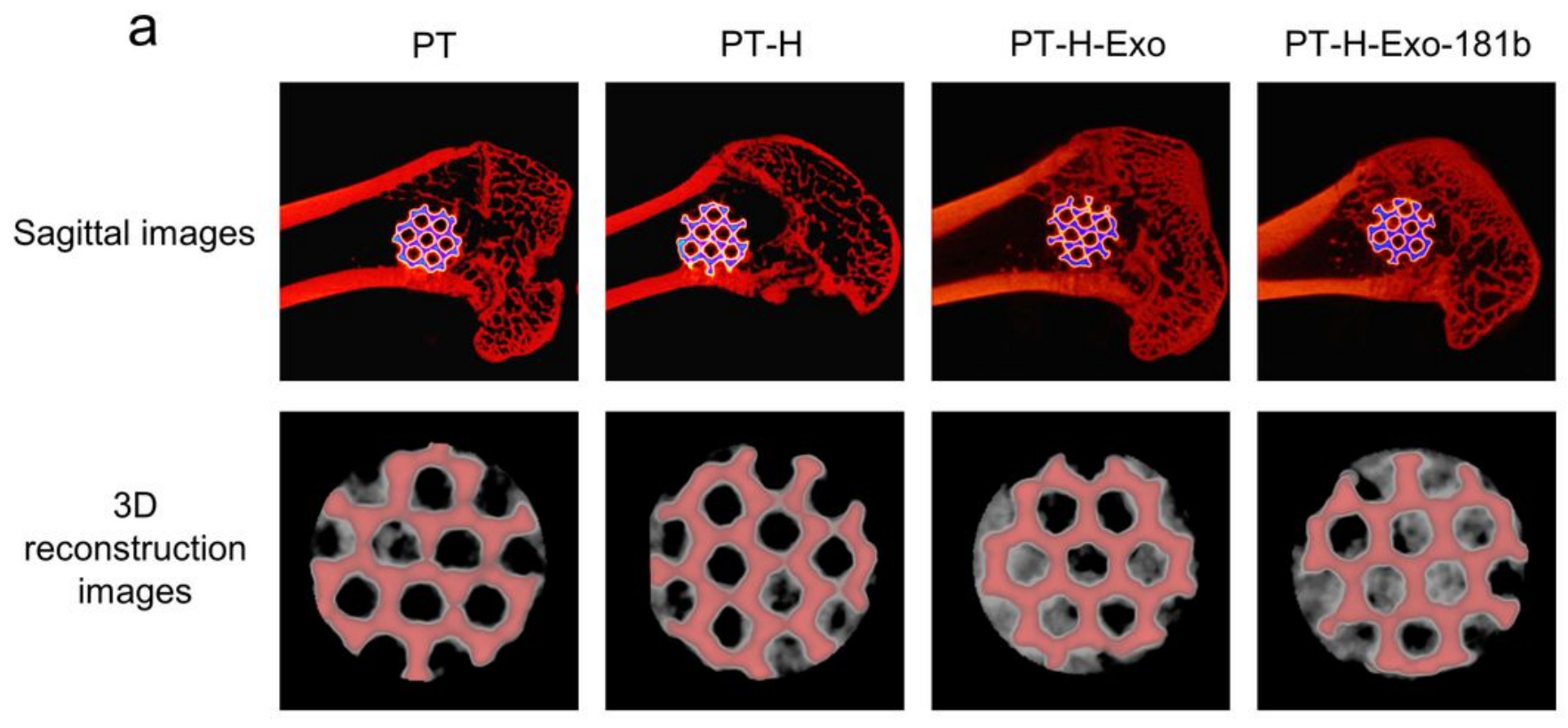

b
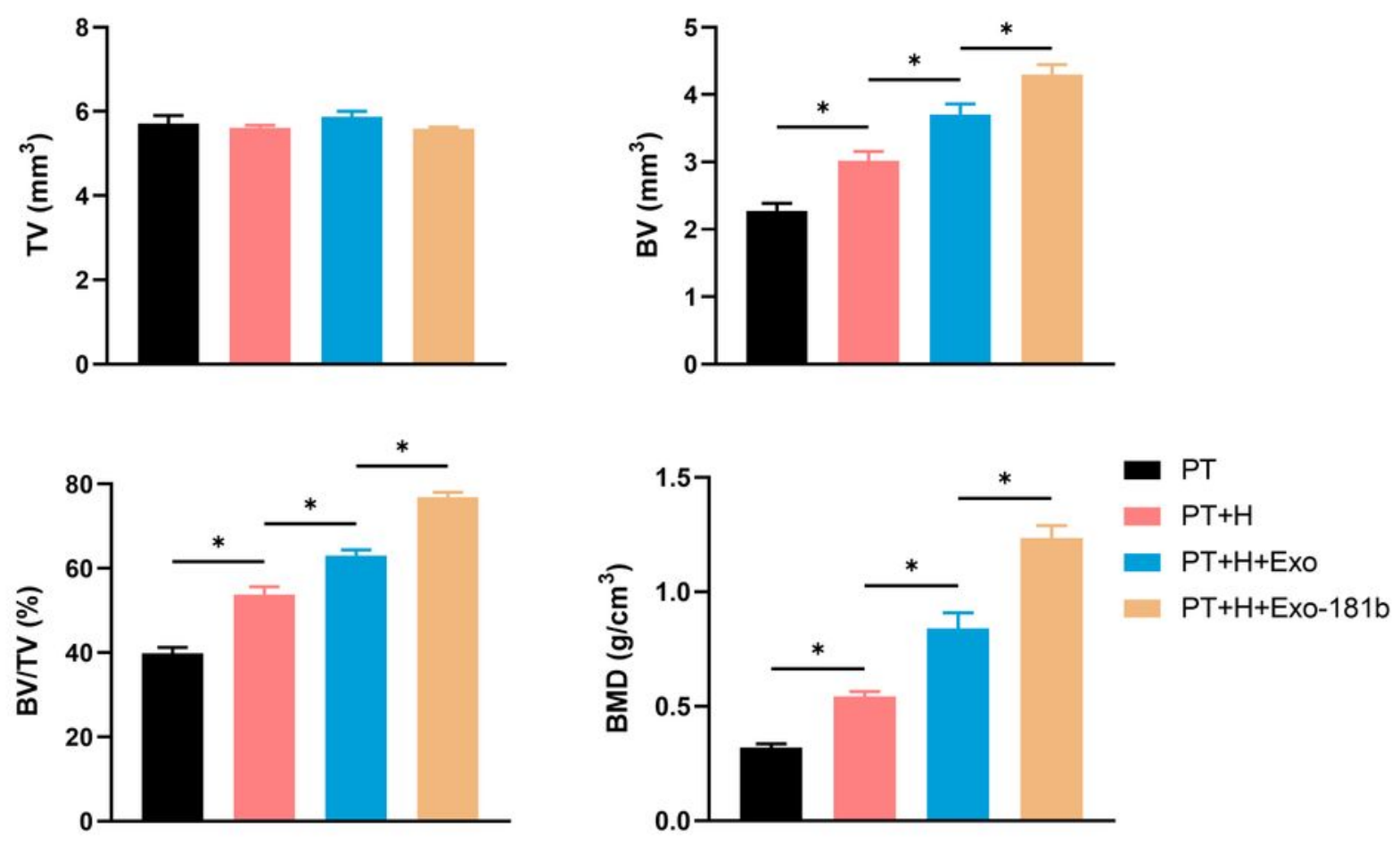

Figure 6

PT-H-Exo-181b promoted osteointegration in vivo detected by MicroCT. a Representative sagittal images and 3D reconstruction of bone defects after 12 weeks treated with porous titanium alloy coated by H-Exo181b via MicroCT after implantation of Ti, Ti-H, Ti-H-Exo and PT-H-Exo-181b scaffolds. b Statistical analysis of TV, BV, BV/TV and BMD by MicroCT results for each group after 12 weeks $(n=3, * P<0.05)$. 


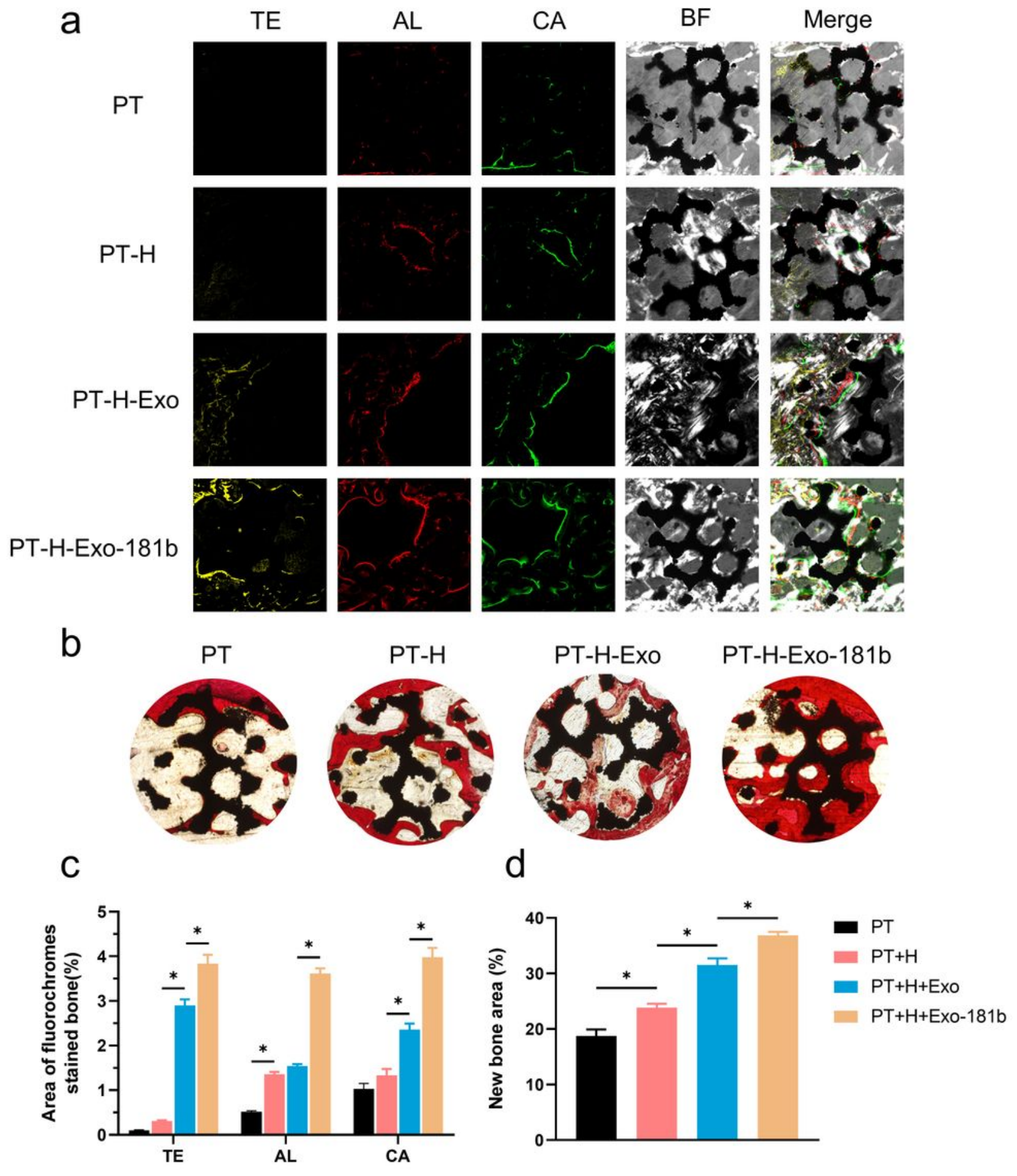

Figure 7

PT-H-Exo-181b promoted osseointegration in vivo detected by histological evaluation. a Fluorochrome labeling analysis was applied for the evaluation of the osteointegration via the undecalcified specimens. The images in yellow, red, green and grey represented tetracycline (TE), alizarinred (AL) calcein (CA) and bright filed (BF) respectively by laser confocal microscopy after the operation. b Van Gieson's picrofuchsin was used for staining of the undecalcified specimens. Newly regenerated bone was stained 
in red. Scale bar $=500 \mu \mathrm{m}$. c Statistical analysis of the area of $T E, A L$ and CA fluorescence. Scale bar $=500$ $\mu \mathrm{m}$. $d$ The percentage area of new regenerated bone was calculated by Image $J(n=3, * P<0.05)$.

a
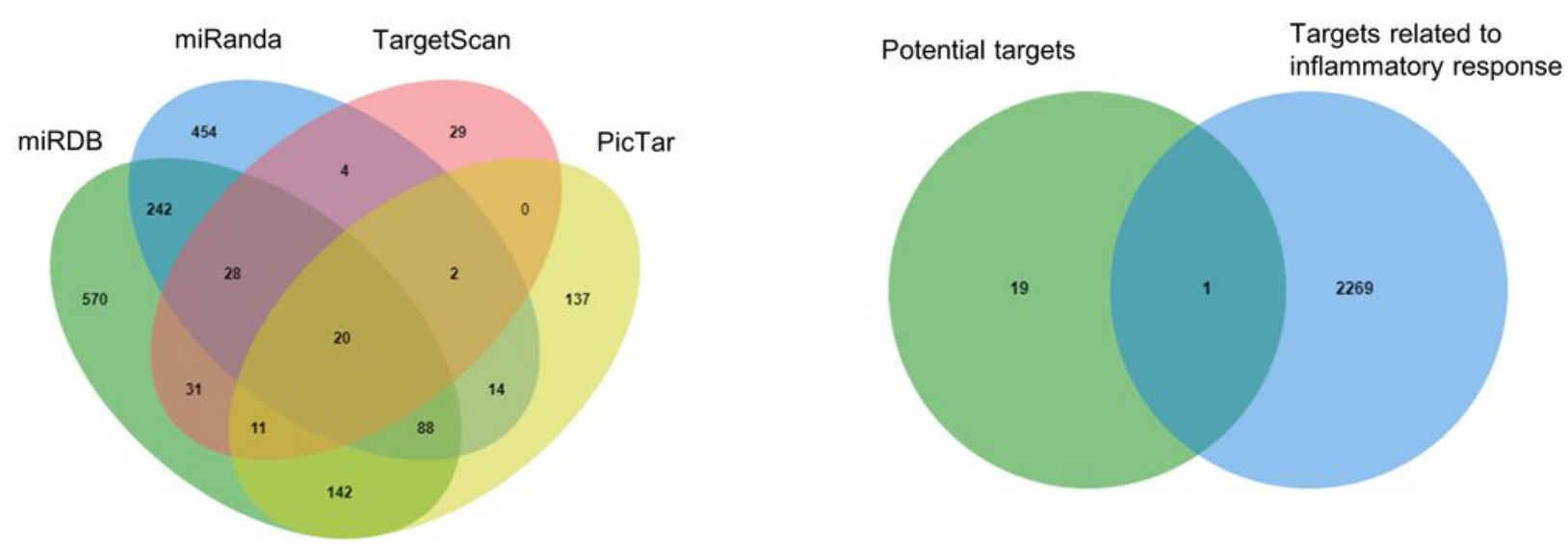

b

C
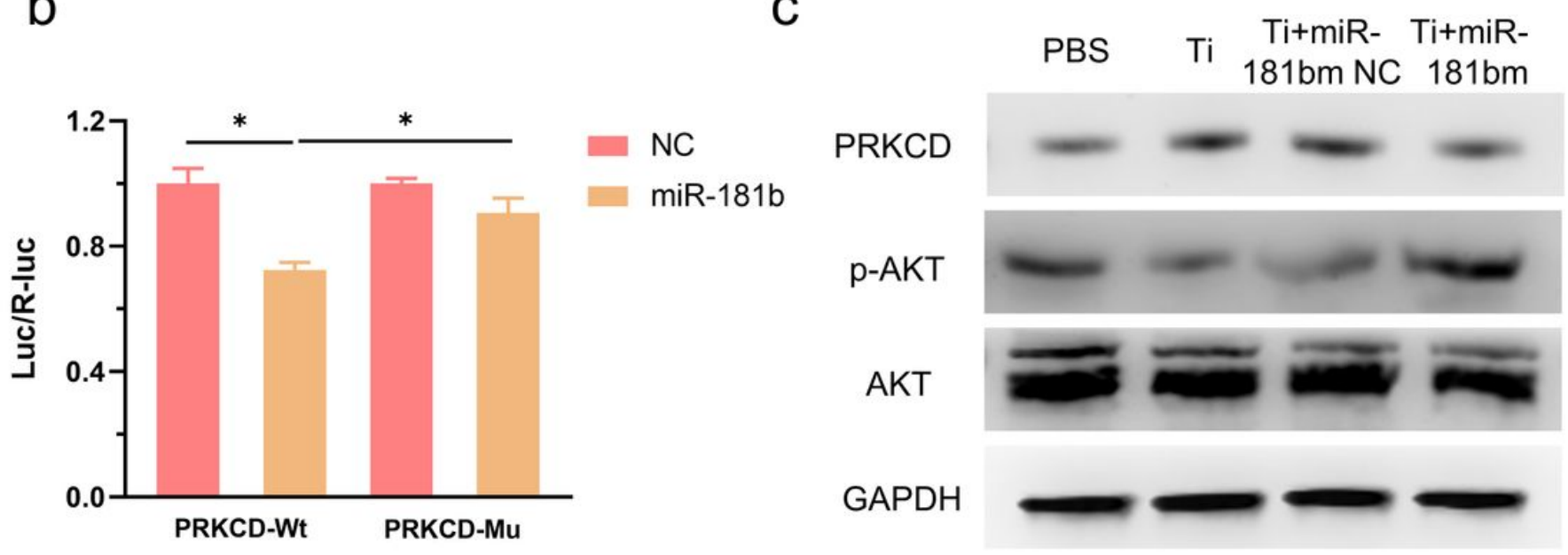

\section{Figure 8}

MiR-181b enhanced M2 polarization by regulating the PRKCD/AKT signaling pathway. a We applied four online prediction tools including TargetScan, miRanda, PicTar and miRDB for the potential targets of miR181b. In addition, we determined the targets with $\mathrm{GO}$ terms related to inflammation response by bioinformatic analysis and the literature. The Venn diagram was used for intersecting the common genes. b The 293T cells were transfected with pRL-CMV reporter vector overexpressing miR-181b, and negative control (NC) was used as the control vector and the direct inhibition of PRKCD by miR-181b were confirmed by dual-luciferase assay. c The expression of PRKCD, p-AKT, AKT, and GAPDH was tested by Western blotting $\left(n=3,{ }^{*}<0.05\right)$. 


\section{a}

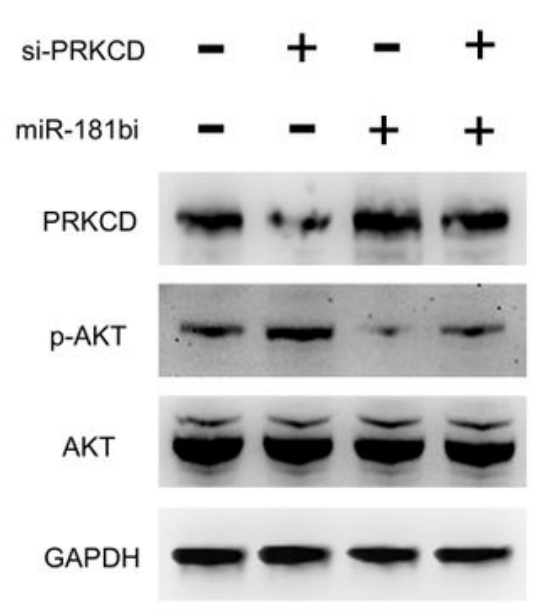

b
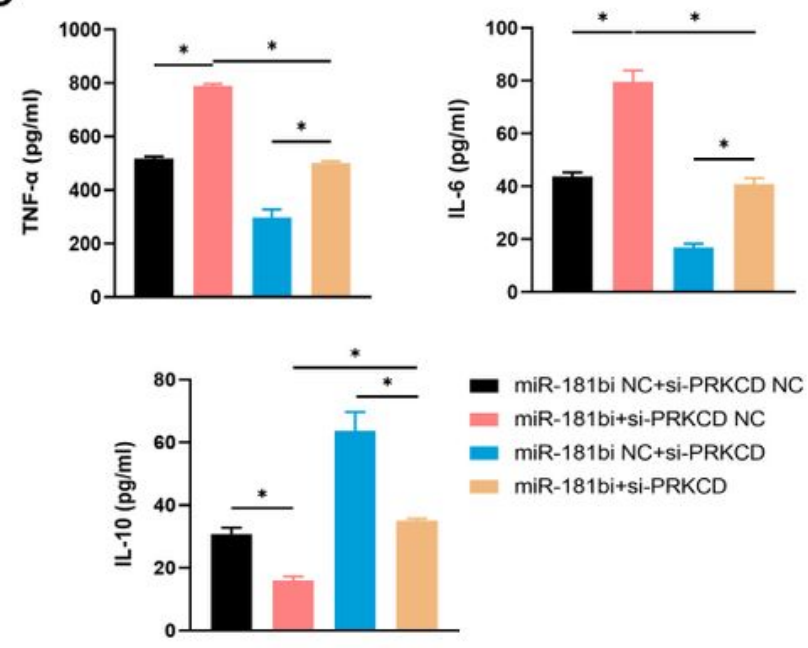

C
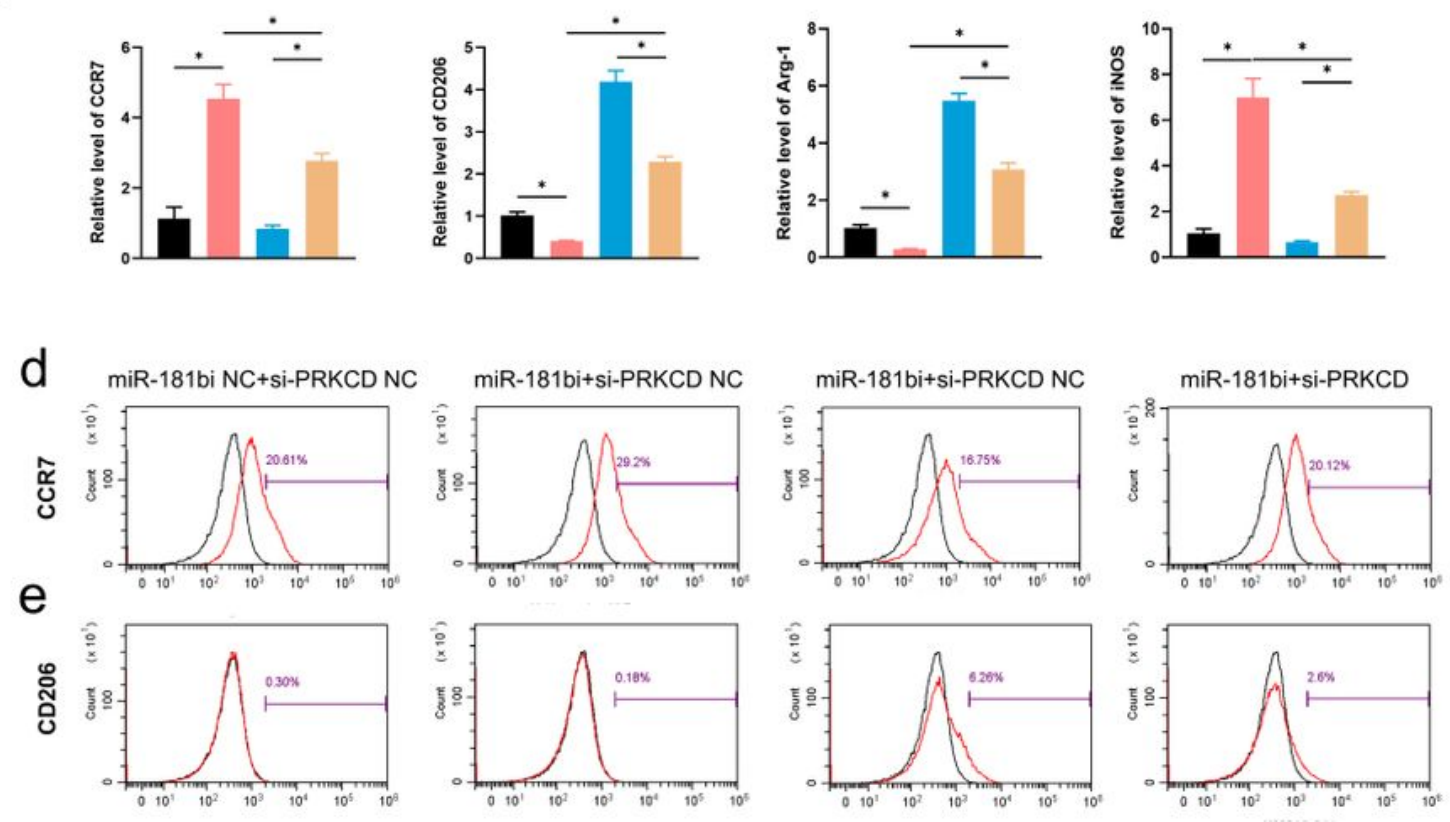

$f$

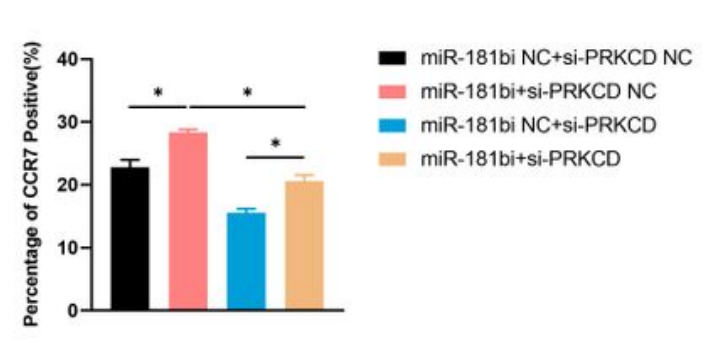

9

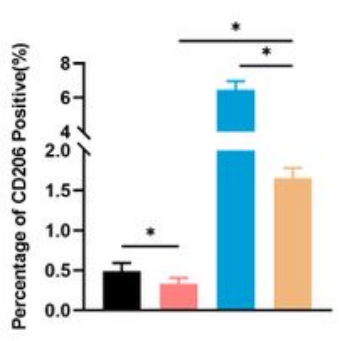

\section{Figure 9}

The inhibition of PRKCD enhanced M2 polarization of miR-181b. a RAW264.7 cells were first treated with Ti and then treated with miR-181bi NC+si-PRKCD NC, miR-181 bi NC+si-PRKCD, miR-181bi +si-PRKCD NC and miR-181bi +si-PRKCD respectively for $24 \mathrm{~h}$. The expression of PRKCD, p-AKT, AKT, and GAPDH was tested by Western blotting. $b$ The concentrations of TNF-a, IL-6, IL-10 of the supernatants by ELISA. $c$ The relative gene expression of CCR7, CD206, Arg-1 and iNOS was detected by qRT-PCR. d-e The percentage 
of CCR7 and CD206 positive cells verified by flow cytometry. f-g The quantitative analysis of the percentage of CCR7 and CD206 positive cells $\left(n=3,{ }^{*} p<0.05\right)$.

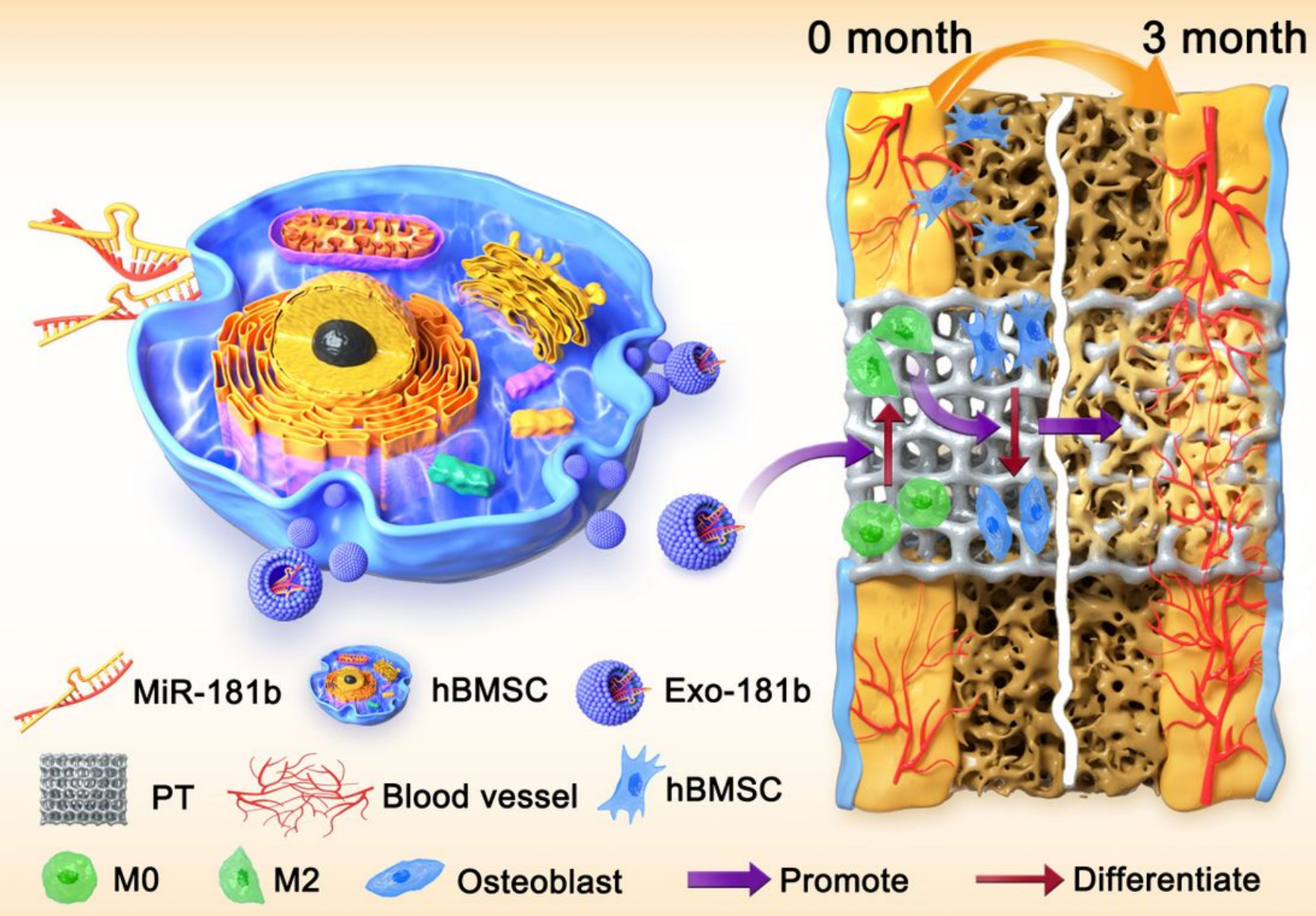

Figure 10

Schematic diagram depicts the detailed mechanisms involved in Exo-181b for osteointegration. Exo-181b promotes the migration and differentiation of hBMSC by promoting macrophage to M2 polarization phenotype, thus promoting the osteogenesis and osteointegration between PT and bone after the implantation in 3 months.

\section{Supplementary Files}

This is a list of supplementary files associated with this preprint. Click to download.

- Primerstable.docx 